\title{
DNA methylation and smoking in Korean adults: epigenome-wide association study
}

Mi Kyeong Lee ${ }^{1,2,3}$, Yoonki Hong ${ }^{3}$, Sun-Young Kim ${ }^{4}$, Stephanie J. London ${ }^{1^{* \dagger}}$ and Woo Jin Kim ${ }^{3^{*+}}$

\begin{abstract}
Background: Exposure to cigarette smoking can increase the risk of cancers and cardiovascular and pulmonary diseases. However, the underlying mechanisms of how smoking contributes to disease risks are not completely understood. Epigenome-wide association studies (EWASs), mostly in non-Asian populations, have been conducted to identify smoking-associated methylation alterations at individual probes. There are few data on regional methylation changes in relation to smoking. Few data link differential methylation in blood to differential gene expression in lung tissue.

Results: We identified 108 significant (false discovery rate (FDR) < 0.05) differentially methylated probes (DMPs) and 87 significant differentially methylated regions (DMRs) (multiple-testing corrected $p<0.01$ ) in current compared to never smokers from our EWAS of cotinine-validated smoking in blood DNA from a Korean chronic obstructive pulmonary disease cohort ( $n=100$ including 31 current, 30 former, and 39 never smokers) using Illumina HumanMethylation450 BeadChip. Of the 108 DMPs (FDR < 0.05), nine CpGs were statistically significant based on Bonferroni correction and 93 were novel including five that mapped to loci previously associated with smoking. Of the 87 DMRs, 66 were mapped to novel loci. Methylation correlated with urine cotinine levels in current smokers at six DMPs, with pack-years in current smokers at six DMPs, and with duration of smoking cessation in former smokers at eight DMPs. Of the 143 genes to which our significant DMPs or DMRs annotated, gene expression levels at 20 genes were associated with pack-years in lung tissue transcriptome data of smokers (Asan Biobank, $n=188$ ).

Conclusions: Our study of differential methylation in Koreans confirmed previous findings from non-Asian populations and revealed novel loci in relation to smoking. Smoking-related differential methylation in blood is associated with gene expression in lung tissue, an important target of adverse health effects of smoking, supporting the potential functional importance of methylation in smoking-related disease.
\end{abstract}

Keywords: DNA methylation, Smoking, Epigenome-wide association study, Cotinine, Duration of smoking cessation, Gene expression

\section{Background}

Smoking is well-known for its adverse health effects [1]; however, between 10 and $35 \%$ of people still smoke daily worldwide [2]. Despite established evidence of the causal relationships between smoking and elevated risk of diseases including cancers [3] and pulmonary [4] and

\footnotetext{
* Correspondence: Iondon2@niehs.nih.gov; pulmo2@kangwon.ac.kr †Equal contributors

${ }^{1}$ Epidemiology Branch, National Institute of Environmental Health Sciences, Department of Health and Human Services, National Institutes of Health, Research Triangle Park, Durham, NC 27709, USA

${ }^{3}$ Department of Internal Medicine and Environmental Health Center, Kangwon National University Hospital, School of Medicine, Kangwon National University, Chuncheon-si, Gangwon-do 19300, South Korea Full list of author information is available at the end of the article
}

cardiovascular diseases [5], the underlying mechanisms are not completely understood. One proposed mechanism is through DNA methylation.

DNA methylation, a type of epigenetic modification, plays a key role in regulating gene expression [6]. Unlike DNA sequence, methylation has cell-type and tissuespecific characteristics. DNA methylation can be impacted by age [7], gender [8], and exposures such as obesity [9] and smoking [10].

At least 16 epigenome-wide association studies (EWASs) of the association between smoking and blood DNA methylation in adults have been published [11-26]. Only one study was conducted in an East Asian population [26]; most have been conducted in populations of European 
ancestry with others in African American, Arab, and South Asian populations. There is no study in Koreans. There are few data where reported smoking has been biochemically validated [11, 21, 25] or where methylation has been evaluated in relation to quantitative biomarkers of smoking $[21,27]$, pack-years, or duration of smoking cessation [22-25]. Only one EWAS correlated differential methylation in blood with gene expression in lung tissue, and only one locus was examined in 10 individuals [19].

The published EWASs of smoking have identified individual differentially methylated probes (DMPs) rather than differentially methylated regions (DMRs). Identification of DMRs associated with an exposure can provide stronger evidence for causality than single DMPs [28]. In addition, DMR analysis is statistically more powerful for detection of association with disease traits or exposures [29].

To identify both DMPs and DMRs in relation to smoking, we conducted an EWAS in 100 adults from a Korean chronic obstructive pulmonary disease (COPD) cohort using the Infinium HumanMethylation450 BeadChip (450k). For the DMPs of genome-wide significance, we investigated their relationship with smoking intensity (urine cotinine) and cumulative smoking (pack-years) in current smokers and duration of smoking cessation in former smokers. As a replication look-up, we also evaluated association between methylation and smoking at previously published probes in our data. For the loci to which significant DMPs or DMRs mapped, we examined differential transcriptome profiles in relation to packyears in lung tissue from a separate population-188 smokers from the Asan Biobank [30].

\section{Methods}

Study participants and exposure to cigarette smoking: the Korean COPD cohort

We aimed to compare methylation in current and former smokers separately to never smokers. For this purpose, we measured DNA methylation in 100 of 190 participants in a Korean COPD cohort [31]. Of the 100 participants, 60 had COPD and 40 were without COPD. The breakdown by smoking was 39 never, 30 former, and 31 current smokers. Subjects were recruited from a rural area in Korea. Having available clinical information, computed tomography (CT) data, survey questionnaire, and blood/urine samples were used for sample selections of methylation profiling. Additional approximate frequency matching on age and smoking status was applied. Details of the COPD cohort have been published [31]. All study participants completed a questionnaire and provided both blood and urine samples. Urine samples were collected at the time of participants' baseline visits. Fresh morning urine samples were obtained from subjects at the time similar with blood sampling. Urine samples had been frozen at $-70{ }^{\circ} \mathrm{C}$. Height $(\mathrm{cm})$ and weight $(\mathrm{kg})$ were measured twice for each participant using a body composition analyzer IOI 353 (Aarna Systems., Udaipur, India); the average value of two measurements was used for further analyses. Body mass index (BMI, $\mathrm{kg} / \mathrm{m}^{2}$ ) was calculated by dividing the weight $(\mathrm{kg})$ by the square of the height $\left(\mathrm{m}^{2}\right)$.

Self-reported smoking status-current, former, and never smoking-was obtained from the questionnaire, and the current status of non-smoking versus smoking was confirmed by urine cotinine levels $(\mathrm{nmol} / \mathrm{L})$ measured by immunoassay (Immulite $2000 \mathrm{Xpi}$; Siemens, NY, USA). One self-reported never smoker was re-assigned to current smoker based on a urine cotinine level of $16,909 \mathrm{nmol} / \mathrm{L}$, higher than our cut-point for current smoking status of $283 \mathrm{nmol} / \mathrm{L}$ [32]. Smokers provided the duration (years) and amount (cigarette packs) of cigarette smoking. Pack-years were calculated by multiplying the number of smoked cigarette packs per day by the number of years smoked. Duration of smoking cessation (years) was reported by former smokers.

\section{Genomic DNA preparation and DNA methylation profiling} We used blood DNA samples from participants' baseline visits for methylation profiling. The DNA quality was checked with a spectrophotometer (NanoDrop ${ }^{\circ}$ ND1000 UV-vis), and genomic DNA was diluted to $50 \mathrm{ng} /$ $\mu \mathrm{l}$ using Quant-iT PicoGreen (Invitrogen, Carlsbad, CA, USA). Bisulfite-conversion using EZ DNA methylation kit (Zymo Research, Irvine, CA, USA) was carried out according to the manufacturer's protocols.

The Infinium HumanMethylation450 BeadChip (Illumina, Inc., San Diego, CA, USA) was used for our genomewide methylation profiling. The methylation value $(\beta)-\mathrm{a}$ ratio between methylated probe intensity and total probe intensity-is interpreted as the proportion of methylation and ranges between 0 (unmethylated) and 1 (methylated). The signal extraction and normalization using Beta MIxture Quantile dilation (BMIQ) [33] were conducted in ChAMP [34]. The ComBat [35] method was applied to adjust for batch effects. Cell-type composition was estimated by Houseman's algorithm [36] in minfi [37]. Cytosinephosphate-guanine $(\mathrm{CpG})$ probe filtering criteria [38] were applied to eliminate sources of possible false positive results, excluding probes that had a detection $p$ value above 0.01 in any sample; had a bead-count less than 3 in $5 \%$ or more of samples; were non-CpG probes; or were nonspecific probes [39]. To minimize the effects of extreme outliers at each probe on association results, methylation values outside three times the interquartile range (IQR) from the first and third quartiles were removed from the analyses. Of all beta values across all participants, 75,549 $(0.19 \%)$ were removed. Probes mapping to the X or Y chromosomes were removed [40]. Therefore, a total of 402,508 CpG probes were used in our EWAS. 


\section{Statistical approach}

We used methylation $\beta$ values because they are more easily interpretable as methylation changes than $M$ values [41] - the $\log 2$ ratio of methylated probe intensity and unmethylated probe intensity. To identify smokingassociated DMPs, we tested methylation levels (response) for association with smoking exposure status (predictor) using robust linear regression. We adjusted for COPD status because of the selection subjects and for age, sex, BMI, and estimated cell-type composition. Never smokers served as the reference group. The regression analysis and empirical Bayes approach was done using Linear Models for Microarray data (limma) [42]. For genome-wide significance, we set the threshold of false discovery rate (FDR) [43] adjusted $p<0.05$. All results in this study are methylation differences in current smokers compared to never smokers unless otherwise noted.

In addition to association analyses at individual probes, we applied two different methods-DMRcate [44] and comb-p [45] - to detect regional methylation alterations. These methods can identify significant DMRs even when there is a lack of genome-wide significance at individual probe level. A DMR does not need to contain a DMP of genome-wide significance. DMRs were calculated based not on raw methylation data but the association results.

The DMR methods work in slightly different ways. DMRcate identifies DMRs using tunable kernel smoothing of association signals across the human genome. We used the "dmrcate" function in the DMRcate R package with an input file containing regression coefficients, standard deviations, and unadjusted $p$ values for each probe from our EWAS of current smoking. In detail, DMRcate re-calculates $p$ values at individual CpGs after modeling the Gaussian smoothing using Satterthwaite [46] method within a predefined bandwidth (the length of a distance), corrects $p$ for multiple-testing, and combines information from nearby significant $\mathrm{CpGs}$ within the bandwidth. In contrast, comb-p identifies regional enrichments of low $p$ values from unevenly spaced $p$ values. It utilizes only unadjusted $p$ values and chromosomal locations at each probe. It performs the Stouffer-Liptak-Kechris (slk) correction to adjust for adjacent $p$ values after calculating auto-correlation, identifies regions of enrichment, generates Stouffer-Liptak regioncorrected $p$ values for each region, and performs Sidak [47] multiple-testing correction.

We defined significant DMRs (1) containing at least two probes, (2) combining information from probes residing within 1000 basepairs (bp), and (3) having multiple-testing corrected $p<0.01$ (FDR for DMRcate and Sidak $p$ for comb-p). These two values-the minimum number of $\mathrm{CpGs}$ in a region and the minimum length of a distance-were the defaults in DMRcate [48], so we used the same values for comb-p to compare results from two approaches. One DMR study using comb-p set the minimum number of probes to 2 and reported DMRs (Sidak $p<0.05$ ) [49]. We used a more strict cutoff for multiple-testing correction (adjusted $p<$ 0.01 ) for statistical significance because these methods have been updated and there is no consensus of the threshold. Relevant parameters for DMR calling can be found in Additional file 1: Table S2. We considered that the same region was identified as differentially methylated by the two methods if the start (bp) or end (bp) site was the same or a region identified by one of the two method resided inside a region identified by the other.

We evaluated whether the genome-wide significant (FDR < 0.05) differential methylation patterns seen in current smokers relative to never smokers were also seen in former compared to never smokers. Therefore, in the former smokers, we adjusted for 108 tests to determine look-up level replication (FDR <0.05). In addition, we examined the dose-response relationships between methylation levels and quantitative indexes of smoking exposure: urine cotinine levels (nmol/L), packyears in current smokers, and time since smoking cessation (years) in former smokers by using the Spearman correlation. For the dose-response analyses, we used nominal statistical significance (unadjusted $p<0.05$ ) to report our findings.

We also examined the association with current smoking for the $192 \mathrm{CpGs}$ reported more than once in the 16 published studies based on either Illumina Infinium HumanMethylation27 BeadChip or 450k array. Of these 192, 178 CpGs were checked for association after probe filtering in our data. The cutoff for statistical significance was set to FDR adjusted $p<0.05$ after correcting for 178 tests.

All statistical analyses were performed in $\mathrm{R}$ (version 3.0.2) [50] except for comb-p [45]. The gene annotation for each probe was based on the manufacturer's annotation file [51].

We used coMET [52] to visualize regional methylation patterns in the top four DMRs (adjusted $p<1.0 \mathrm{E}-10$ at both analyses). In addition to gene names and regulatory elements of the region from ENSEMBLE, Digital DNaseI Hypersensitivity Clusters from ENCODE (DNase Cluster) and chromatin state segmentation by HMM from ENCODE/Broad (Broad ChromHMM) were added (Additional file 2: Figure S2).

\section{Enrichment and functional network analysis}

We performed an enrichment analysis to examine whether the significant DMPs (FDR < 0.05) were overor under-represented, compared to all probes from the $450 \mathrm{k}$ array, in several biological features from the 
Illumina annotation file. The hypergeometric test (twosided doubling mid-p) was used for the evaluation of enrichments or depletions.

For biological insights into differential methylation changes in relation to current smoking, we implemented a functional network analysis. Genes annotated from selected DMPs (FDR < 0.10) were included in the analysis. We used a core analysis of Ingenuity Pathway Analysis (Ingenuity Systems, Inc., Redwood City, CA, USA).

\section{Transcriptome analysis: Asan Biobank}

Transcriptome profiles from the lung tissues of 188 male smokers from the Asan Biobank were used in this analysis. Details of transcriptome profiles using RNA-seq (HiSeq 2000 system, Illumina, Inc., San Diego, CA, USA) have been published [30]. Data was available at NCBI Gene Expression Omnibus (GEO) (accession number of GSE57148). To exclude potential impact of extreme values, we filtered gene expression values outside of three times the IQR from the first and third quartiles of each gene transcript. Of all gene expression values across all participants, 35,607 (1.1\%) were removed. We calculated pack-years from duration (years) and amount (cigarette packs) of cigarette smoking.

To identify differentially expressed genes in relation to smoking intensity (pack-years), we applied a robust linear regression model and empirical Bayes approach by using limma [42]. For robust linear regression, gene expression levels were the response and pack-years the predictor. We presented nominally significant results to provide a clue to understand relationships between methylation in blood and gene expression in lung tissue.

\section{Results}

The descriptive characteristics of the study populations are shown in Table 1 . The study participants were aged 53 to 84 years. There were 39 never, 30 former, and 31 current smokers. Among the never smokers, 6 were male and 33 were female. The former smokers were all male. There was one female current smoker. Individuals diagnosed with COPD were represented in each smoking group as follows: 19 in never, 20 in former, and 21 in current smoking group. The average BMI was $23.2 \mathrm{~kg} /$ $\mathrm{m}^{2}$ for never smokers, $23.5 \mathrm{~kg} / \mathrm{m}^{2}$ for former smokers, and $22 \mathrm{~kg} / \mathrm{m}^{2}$ for current smokers. The duration of smoking cessation in former smokers ranged 7 to 40 years. There were no significant differences in age, BMI, and proportion of COPD cases across smoking groups in our EWAS data.

We identified 108 significant DMPs in current smokers compared to never smokers (FDR <0.05) (Table 2, Additional file 3: Table S1, and Additional file 4: Table S3). Of these, nine were significant after Bonferroni correction (unadjusted $p<1.2 \mathrm{E}-07$ correcting for 402,508 tests). Of the FDR-significant DMPs, 93 of these were novel and 15 were previously reported in EWASs of smoking. Decreased methylation in current smokers was observed at $85 \%$ of the significant DMPs. The methylation differences between current and never smokers at significant CpGs ranged from -20.3 to $15.6 \%$. Among the top five probes, the most highly statistically significant was a $\mathrm{CpG}$ well-known for its association with smoking: $\operatorname{cg} 05575921$ $(\mathrm{FDR}=2.6 \mathrm{E}-07)$ in aryl-hydrocarbon receptor repressor $(A H R R)$. Among the remaining four probes in the top five, three were novel-cg10664184 $(\mathrm{FDR}=1.80 \mathrm{E}-05)$ in DDA1; $\operatorname{cg} 20723792$ (FDR $=6.40 \mathrm{E}-05)$ in FAM53B; and $\operatorname{cg} 24780263(\mathrm{FDR}=0.001)$ in $A L D O A$-except for cg05951221 (FDR $=8.50 \mathrm{E}-04)$ located 12,850 base pair (bp) apart from ALPPL2. At five loci, more than one DMP at genome-wide significance was identified: $A H R R$ (3 probes), 2q37.1 near ALPPL2 (2 probes), MYO1G (2 probes), NKX2-3 (2 probes), and FAM82A2 (2 probes). The genomic inflation factor (lambda) was 1.25. Manhattan plot and QQ plot are provided (Additional file 5: Figure S1).

For our 108 significant DMPs, we found enrichment of probes mapping to $\mathrm{CpG}$ island shores (35 versus $23 \%$ overall from the array, $p=0.002$ ) and enhancer (29 versus $21 \%$ overall from the array, $p=0.04$ ). No significant over- or under-representation of probes in promoterassociated regions (19 versus $19 \%$ overall, $p>0.05$ ) or DNase hypersensitivity sites (18 versus $12 \%$ overall, $p>$ 0.05) were detected.

From the two different DMR analyses, we discovered 249 significant $(\mathrm{FDR}<0.01)$ DMRs from DMRcate, 102 significant (Sidak $p<0.01$ ) DMRs from comb-p, and 87 significant based on both approaches (Table 3). Of these 87 significant using both methods, 66 regions were novel, meaning never reported in previous EWASs of smoking in adults, including 7 that contained one of our genome-wide significant individual DMPs. Among those 87 DMRs, the most significant one (chromosome:start position-end position) from DMRcate was chr5:373378$374425(\mathrm{FDR}=4.6 \mathrm{E}-17)$ in $A H R R$ and this region contains five probes-cg05575921, cg22103736, cg08714121, cg04141806, and cg22356527-including our top-ranked DMP. AHRR differential methylation was also observed from comb-p with two probes-cg05575921 and cg22103736-in slightly shorter length (chr5:373378373887; Sidak $p=4.8 \mathrm{E}-05)$ than that from DMRcate. The most significant DMR overall from comb-p was chr6:149805995-149806732 (Sidak $p=1.9 \mathrm{E}-14$ ) in $Z C 3 H 12 D$ and the exact same region, meaning the same start, end, and number of probes, was also observed from DMRcate (FDR $=2.3 E-15)$ (Table 3). This region did not contain a genome-wide significant DMP. Among novel DMRs, the top two regions from both analyses were chr4:81117647-81119473 (FDR = 6.7E-13 from DMRcate; 
Sidak $p=2.9 \mathrm{E}-13$ from comb-p) at PRDM8 including 11 probes and chr4:103940711-103941300 (FDR = 6.8E-14 from DMRcate; Sidak $p=2.7 \mathrm{E}-10$ from comb-p) at $S L C 9 B 1$ including 11 probes. Details of the top five DMRs from each software are in Additional file 6: Table S4. Those regions contain either one or two highly significant CpGs or tightly spaced CpGs of nominal statistical significance. The average (standard deviation, SD) of distances of nearby CpGs in those regions was 147 (153) bp for DMRcate and 158 (169) bp for comb-p.

Among the 108 significant DMPs from the comparison of current to never smokers, 104 were also significant in the former to never smoker comparison (FDR $<0.05$, look-up level replication) and had effects in the same direction (Additional file 7: Table S5). The attenuation in effect size in former compared with current smokers ranged from -12.3 to $4.3 \%$. The top-ranked DMP in former smokers compared to never smokers was cg20723792 (FDR = 1.3E-2) in FAM53B at which no relationship with smoking exposures in terms of DNA methylation has been previously reported.

We examined dose-response relationships between methylation levels and quantitative measures of smoking exposure (urine cotinine levels and pack-years in current smokers and duration of smoking cessation in former smokers) for the 108 significant DMPs identified in our EWAS of current smoking (Table 4). There was no significant finding after FDR multiple-testing correction. Urine cotinine levels were positively correlated at nominal levels of significance (uncorrected $p<0.05$ ) with methylation levels at a probe in MTNR1A and negatively correlated with methylation levels at five probes from five different loci: GNG12; GPR15; AHRR; FAM82A2; and F2RL3. Packyears in current smokers showed positive correlation at five loci and negative correlation with methylation levels at one locus. Duration of smoking cessation in former smokers was positively correlated at nominal significance $(p<0.05)$ with methylation levels at seven loci and negatively correlated with methylation at one locus.

Our analysis of differential gene expression in lung tissue was conducted in 188 male smokers from a separate study, the Asan Biobank. The average age was 64.2 (SD = 8.7) years and average pack-years was $42.0(\mathrm{SD}=20.6)$ (Table 1). Of the 174 genes to which the 108 DMPs or 87 DMRs that were significantly differentially methylated were annotated, we had gene transcript profiles for 143 . Of these, 20 genes, annotated from 17 DMPs or eight DMRs, showed nominally significant differential gene expression profiles $(p<0.05)$ in relation to pack-years (Table 5). Fourteen of the 20 genes were novel loci for effects of smoking on methylation and six-GPR15, AHRR, ELMO1, SNED1, LPP, and GNA12-were previously reported in EWASs of smoking. No significant results were observed after FDR multiple-testing correction.
In current smokers compared to never smokers, there were lower methylation levels at 17 DMPs (Table 5). Of those, four CpGs were located in enhancer regions and their corresponding lung tissue gene expression values were positively associated with pack-years in smokers, regardless of whether or not they were located in a CpG island. Four of the 17 were at DNase I hypersensitivity sites (DHS). Three of these were outside of CpG islands and showed a positive association with pack-years in smokers. The remaining site, located on a shelf region of a CpG island, was negatively associated. At four promoter-associated CpGs, we did not find any relationships between methylation levels and gene expression values.

Our functional network mapping involving 221 genes annotated from probes in our EWAS (FDR <0.10) identified four overrepresented pathways (Additional file 8: Table S6). Top three networks were "gene expression, cellular movement, and embryonic movement," "cancer, cellular development, organismal injury, and abnormalities," and "hematological, metabolic, and cardiovascular disease."

From a replication look-up of 178 CpGs, selected based on significant findings in at least two published EWASs of smoking, we confirmed differential methylation at $70 \mathrm{CpGs}$ (Table 6). Of these, all CpGs showed same direction of association compared to that in previous reports. Among these 178 probes from previous EWASs, $83(47 \%)$ showed nominal $(p<0.05)$ association in our analysis of current smokers which is much higher expected by chance (Kolmogorov $p<2.2 \mathrm{E}-16$ ). There were also significant differential methylation changes in former smokers at 24 CpGs in 17 loci (Table 6).

\section{Discussion}

This is the second EWAS for smoking exposure in an East Asian population and the first which links differential methylation changes in blood to large-scale differential transcriptome profiles in lung tissue at multiple loci. We discovered novel smoking-associated DMRs as well as DMPs and confirmed previous findings mostly from non-Asian populations. We identified nominally significant correlations in DNA methylation in relation to quantitative measures of smoking: urine cotinine levels, pack-years, and duration of smoking cessation. Differentially expressed genes in relation to smoking intensity in lung tissue support the potential utility of our findings as blood DNA methylation biomarkers for smoking exposure.

We discovered 108 significant DMPs and 87 significant DMRs in relation to current smoking. Fourteen loci were significant from both approaches; nine of which were novel: CALML4, CCND1, FOXK2, LINC01019, NKX2-3, NT5C1A, PRDM8, SPAG17, and SYNGR1. It 
Table 1 Descriptive characteristics of the study population

\begin{tabular}{|c|c|c|c|c|}
\hline \multirow{2}{*}{$\begin{array}{l}\text { Characteristics (mean } \pm \text { standard } \\
\text { deviation or } n(\%))\end{array}$} & \multicolumn{3}{|c|}{ Genome-wide methylation analysis in blood DNA (the Korean COPD cohort) } & \multirow{2}{*}{$\begin{array}{l}\text { Transcriptome analysis in } \\
\text { lung tissue (Asan Biobank) }\end{array}$} \\
\hline & Never smoker $(N=39)$ & Former smoker $(N=30)$ & Current smoker $(N=31)$ & \\
\hline Male & $6(15.4)$ & $30(100)$ & $30(96.8)$ & $188(100)$ \\
\hline Female & $33(84.6)$ & $0(0)$ & $1(3.2)$ & $0(0)$ \\
\hline Age, years & $72.9 \pm 6.1$ & $74.1 \pm 7.4$ & $71.5 \pm 5.3$ & $64.2 \pm 8.7$ \\
\hline Body mass index, $\mathrm{kg} / \mathrm{m}^{2}$ & $23.2 \pm 3.0$ & $23.5 \pm 2.7$ & $22 \pm 2.8$ & NA \\
\hline Pack-year & $N A^{c}$ & $28.9 \pm 19.6$ & $35.7 \pm 19.1$ & $42.0 \pm 20.6$ \\
\hline Duration of smoking cessation, years & NA & $17.6 \pm 7.5$ & NA & NA \\
\hline Urine cotinine, $\mathrm{nmol} / \mathrm{L}$ & $88.4 \pm 3.2^{d}$ & $167.6^{e}$ & $29421 \pm 21947$ & NA \\
\hline Undetectable $e^{a}$ & $36(92.3)$ & $29(96.7)$ & $0(0)$ & NA \\
\hline $\mathrm{COPD}^{\mathrm{b}}$ & $19(48.7)$ & $20(66.7)$ & $21(67.7)$ & $98(51.9)$ \\
\hline
\end{tabular}

aUrine cotinine levels $\leq 56.8 \mathrm{nmol} / \mathrm{L}$ are marked as "undetectable" from the measurement using IMMULITE 2000 Immunoassay System (Siemens Healthcare Diagnostics Inc., Tarrytown, NY, USA)

${ }^{\mathrm{b}}$ Chronic obstructive pulmonary disease

cNot available

${ }^{d}$ Urine cotinine levels in three never smokers were detectable

e Urine cotinine level in only one former smoker was detectable and the level was $167.6 \mathrm{nmol} / \mathrm{L}$

has been reported that genetic variants in CCND1 and smoking exposure are associated with gastric carcinogenesis [53], nasopharyngeal carcinoma [54], and lung cancer [55] and useful for lung cancer prediction [56]. PRDM8 encodes a protein which belongs to a conserved family of histone methyltransferases regulating transcription negatively.

Of the 87 significant DMRs, in 32 all CpGs were of nominal $(p<0.05)$ statistical significance. On average, $78 \%$ of CpGs in each identified DMR were nominally significant. Although a DMR does not need to include a genome-wide significant DMP in the region, 14 DMRs contained FDR-significant DMPs. In our analysis of differentially methylated regions, the most highly significant DMRs consist of either one or two highly significant DMPs or closely spaced neighboring CpGs of only nominal statistical significance in the region (Additional file 6: Table S4). Although it has been reported that two methods that we used to identify DMRs can correct for irregular spacing of probes across the genome [44, 45], we cannot conclude whether these are reflecting true differential methylation or false discovery driven by array-design.

Our EWAS identified 104 DMPs from the analysis of current smokers that were also seen in former smokers compared to never smokers; 93 of which were novel. The methylation differences in current and former smokers compared to never smokers were only slightly attenuated. The persistence of blood DNA methylation changes in former smokers, even after 7 to 40 years of smoking cessation, is notable. Our analysis of duration of smoking cessation in former smokers showed positive correlations at seven loci-IFI16, CLASP1, KTELC1, SPEF2, ACOT13, BSPRY, and FAM82A2-which has not been previously reported in EWASs. We also found a negative correlation at cg25799109 in ARHGEF3, a known smoking-associated CpG [12].

Although there are biomarkers of current smoking, including nicotine and its metabolite cotinine levels in urine, blood, or saliva, biomarkers reflecting past smoking have been lacking. Interestingly, we found that most of the signals for current smoking remained for past smoking. Recent studies suggest that methylation signals are promising biomarkers for both current and lifetime smoking [57] that are related to mortality [58]. Significant methylation alterations in former smokers compared to never smokers from our study can contribute to development of biomarkers for past smoking.

For urinary cotinine, we confirmed previous findings of differential methylation at GNG12, GPR15, F2RL3 [27], and $A H R R[21,27]$ at nominal statistical significance $(p<$ 0.05 ) and negative directions of association were also consistent. We also identified novel positive and negative correlations with methylation levels at MTNR1A and FAM82A2, respectively. Gene-environment interactions of variants in MTNR1A and smoking have been reported in relation to oral cancer [59]. In studies without cotinine measured, differential methylation at loci correlated with cotinine could serve as objective biomarkers to confirm the self-reported current level of smoking. For pack-years, we found correlations with DNA methylation at NT5C1A, ZBTB9, HPX, CCND1, and RNF160 which were have not been reported in previous EWASs. Although cg19134728 in JAKMIP3 was previously shown to be differentially methylated in smokers compared to non-smokers [15], its relationship with pack-years in current smokers was never studied.

To gain some biological insight into the differential methylation from our EWAS, we linked our genomewide significant results to large-scale transcriptome profiles in lung tissues. We discovered differential gene 
Table 2 Top 30 CpGs differentially methylated in blood DNA in relation to current smoking compared to never smoking (FDR $<0.05$, ordered by chromosomal location)

\begin{tabular}{|c|c|c|c|c|c|c|c|}
\hline $\mathrm{Chr}^{\mathrm{a}}$ & Gene & Distance to gene ${ }^{b}$ & Probe & Position $^{c}$ & Coef $^{d}$ & $\mathrm{SE}^{\mathrm{e}}$ & $P^{f}$ \\
\hline \multirow[t]{5}{*}{2} & CCDC104 & & cg21597209 & 55746709 & -0.009 & 0.002 & $6.2 \mathrm{E}-07$ \\
\hline & DGUOK & & cg19394739 & 74154363 & -0.012 & 0.002 & $3.5 \mathrm{E}-07$ \\
\hline & CLASP1 & & $\operatorname{cg} 22346073$ & 122402890 & -0.056 & 0.010 & $5.1 \mathrm{E}-08$ \\
\hline & SATB2 & & cg21136715 & 200322252 & -0.035 & 0.006 & $2.1 \mathrm{E}-07$ \\
\hline & ALPPL2 & 12,850 & $\operatorname{cg} 05951221^{9}$ & 233284402 & -0.088 & 0.014 & $8.4 \mathrm{E}-09$ \\
\hline 3 & GPR15 & & cg198592709 & 98251294 & -0.027 & 0.005 & $1.0 \mathrm{E}-07$ \\
\hline \multirow[t]{4}{*}{5} & AHRR & & $\operatorname{cg} 05575921^{9}$ & 373378 & -0.203 & 0.025 & $6.5 E-13$ \\
\hline & & & cg256482039 & 395444 & -0.079 & 0.015 & $6.2 \mathrm{E}-07$ \\
\hline & LINC01019 & $-239,389$ & cg11405538 & 3177877 & 0.124 & 0.022 & $1.3 \mathrm{E}-07$ \\
\hline & $50 \times 30$ & & cg06995810 & 157079468 & 0.048 & 0.009 & $1.0 \mathrm{E}-06$ \\
\hline \multirow[t]{3}{*}{7} & TSPAN13 & & cg05848863 & 16794078 & -0.024 & 0.004 & $3.6 \mathrm{E}-07$ \\
\hline & PLEKHAB & & cg09762120 & 30108301 & 0.040 & 0.007 & $2.8 \mathrm{E}-08$ \\
\hline & ADCYAPIR1 & & cg20165074 & 31091813 & -0.008 & 0.002 & $6.7 \mathrm{E}-07$ \\
\hline 10 & FAM53B & & cg20723792 & 126360669 & -0.097 & 0.014 & 4.8E-10 \\
\hline \multirow[t]{4}{*}{11} & IRF7 & & cg27271532 & 612762 & -0.035 & 0.006 & $3.8 \mathrm{E}-07$ \\
\hline & E2F8 & & cg15604507 & 19263433 & -0.021 & 0.004 & 5.7E-07 \\
\hline & CCND1 & & cg09520904 & 69462943 & -0.036 & 0.007 & $7.5 \mathrm{E}-07$ \\
\hline & $D I X D C 1$ & & $\operatorname{cg} 11471799$ & 111807548 & -0.023 & 0.004 & $6.2 \mathrm{E}-07$ \\
\hline 12 & $C D K 2 A P 1$ & & cg13421247 & 123756945 & -0.058 & 0.011 & $9.8 \mathrm{E}-07$ \\
\hline \multirow[t]{2}{*}{14} & $C F L 2$ & $-44,147$ & cg23429457 & 35135441 & -0.040 & 0.007 & $2.0 \mathrm{E}-07$ \\
\hline & EXOC3L4 & $-20,369$ & cg04884342 & 103546112 & 0.020 & 0.004 & $5.6 \mathrm{E}-07$ \\
\hline \multirow[t]{3}{*}{15} & CALML4 & & cg00388154 & 68498857 & -0.058 & 0.011 & $2.9 \mathrm{E}-07$ \\
\hline & $\mathrm{CORO} 2 \mathrm{~B}$ & & cg18765659 & 69018349 & -0.053 & 0.010 & 7.4E-07 \\
\hline & TLE3 & & cg06730438 & 70355664 & -0.016 & 0.003 & $4.9 \mathrm{E}-07$ \\
\hline \multirow[t]{2}{*}{16} & ALDOA & & cg24780263 & 30064201 & -0.011 & 0.002 & $1.8 \mathrm{E}-08$ \\
\hline & KIAA0182 & & cg26723054 & 85650522 & -0.038 & 0.007 & 7.2E-07 \\
\hline \multirow[t]{3}{*}{19} & $F 2 R L 3$ & & cg036361839 & 17000585 & -0.128 & 0.021 & $2.0 \mathrm{E}-08$ \\
\hline & DDA1 & & cg10664184 & 17420304 & -0.028 & 0.004 & $9.2 \mathrm{E}-11$ \\
\hline & CD33 & & cg06861672 & 51727798 & -0.036 & 0.007 & $3.3 \mathrm{E}-07$ \\
\hline 21 & MIR155HG & & cg03872783 & 26934885 & -0.008 & 0.001 & $9.7 \mathrm{E}-07$ \\
\hline
\end{tabular}

${ }^{a}$ Chromosome

${ }^{b}$ Distance to transcription start site of the mapped gene (basepair)

${ }^{c}$ Physical position (basepair, National Center for Biotechnology Information human reference genome assembly Build 37.3)

${ }^{\mathrm{d}}$ Regression coefficient from statistical model

estandard error of regression coefficient

f Statistical significance from statistical model

${ }^{9}$ Probe identified in previous epigenome-wide association studies (EWASs) of smoking

${ }^{h}$ Probe mapped to genes identified in previous EWASs of smoking

DMPs ordered by $p$ values can be found in Additional file 10

expressions in relation to pack-years at 20 genes which were mapped from 17 DMPs and 8 DMRs. Our findings include six genes-GPR15, AHRR, LPP, GNA12, $C Y B 561$, and SNED1-known for their association with smoking in previous EWASs, but none of these has been identified in transcriptome analyses of pack-years in lung tissue. Only one previous EWAS included smokingassociated differential gene expression at $A H R R$; that study included lung tissue samples from five smokers and five non-smokers [19].

Our finding of enrichment of significant DMPs in CpG island shore (regions within 2000 bp within a CpG island) is consistent with previous findings of variable DNA methylation in the regions [60], suggesting methylation in shore regions is more susceptible to environmental factors including smoking. 
Table 3 Differentially methylated regions in blood DNA in relation to current smoking compared to never smoking (multiple-testing corrected $p<0.01$ at DMRcate and comb-p, ordered by chromosomal location)

\begin{tabular}{|c|c|c|c|c|c|c|c|c|c|c|c|}
\hline \multirow[t]{2}{*}{$\overline{\mathrm{Chr}^{\mathrm{a}}}$} & \multirow[t]{2}{*}{ Gene } & \multirow{2}{*}{$\begin{array}{l}\text { Distance } \\
\text { to gene }\end{array}$} & \multicolumn{4}{|l|}{ DMRcate } & \multicolumn{4}{|l|}{ Comb-p } & \multirow{2}{*}{$\begin{array}{l}\text { Minimum } \\
P^{i}\end{array}$} \\
\hline & & & Start $\left(b^{c}\right)$ & End (bp) & $\mathrm{FDR}^{\mathrm{d}}$ & $\# \mathrm{CpGs}^{\mathrm{e}}$ & Start (bp) & End (bp) & Sidak $P^{f}$ & $\overline{\# C p G s}$ & \\
\hline \multirow[t]{11}{*}{1} & MXRA8 & -812 & 1286917 & 1287259 & 0.002 & $2(2)$ & & & 0.002 & & $2.2 \mathrm{E}-04$ \\
\hline & CASZ1 & -600 & 10695686 & 10696066 & 8.7E-04 & $2(2)$ & & & 0.009 & & $1.5 \mathrm{E}-05$ \\
\hline & $A H D C 1$ & & 27929092 & 27929260 & $2.2 \mathrm{E}-04$ & $2(2)$ & & & 0.006 & & $1.5 \mathrm{E}-04$ \\
\hline & NT5C1A & & $40137636^{9}$ & 40138402 & $3.2 \mathrm{E}-06$ & $6(3)$ & & & 0.001 & & $5.5 \mathrm{E}-06$ \\
\hline & ACOT11 $1^{\mathrm{h}}$ & $-58,441$ & 54954187 & 54955366 & 0.002 & $7(4)$ & 54953632 & & 0.009 & $8(4)$ & $6.1 \mathrm{E}-04$ \\
\hline & GNG12h & & $68298816^{9}$ & 68299511 & $7.0 \mathrm{E}-07$ & $7(5)$ & 68299057 & & 0.001 & $6(5)$ & $1.4 \mathrm{E}-06$ \\
\hline & $G F / 11^{\text {h }}$ & & 92946700 & 92947961 & $1.1 \mathrm{E}-04$ & $6(4)$ & & & $1.2 \mathrm{E}-04$ & & 8.1E-05 \\
\hline & SPAG17 & & $118727658^{9}$ & 118728226 & $1.3 \mathrm{E}-04$ & $10(2)$ & & & 0.005 & & 7.1E-06 \\
\hline & ZNF697 & & 120173989 & 120174570 & 0.006 & $4(4)$ & & 120174873 & 0.006 & $6(4)$ & 0.002 \\
\hline & GALNT2 & & 230415343 & 230416101 & 0.002 & $6(3)$ & 230414987 & 230417096 & $1.2 \mathrm{E}-04$ & $12(4)$ & 0.005 \\
\hline & SCCPDH & $-26,962$ & 246859889 & 246860416 & 7.0E-04 & $5(4)$ & & & $2.6 \mathrm{E}-04$ & & 0.002 \\
\hline \multirow[t]{3}{*}{2} & PAX8 & & 113992762 & 113993313 & 0.005 & $8(6)$ & & & 0.004 & & 0.011 \\
\hline & $A L P P L 2^{h}$ & 11,458 & $233283010^{9}$ & 233285607 & $8.0 \mathrm{E}-15$ & $8(5)$ & & & $1.5 \mathrm{E}-13$ & & 8.4E-09 \\
\hline & SNED $1^{\text {h }}$ & & 241975756 & 241976244 & $1.9 \mathrm{E}-06$ & $4(4)$ & & & $3.8 \mathrm{E}-06$ & & $1.4 \mathrm{E}-04$ \\
\hline \multirow[t]{5}{*}{3} & KRBOX1 & 11 & 42977777 & 42978180 & 9.7E-04 & $7(5)$ & & & 0.003 & & 4.1E-04 \\
\hline & GPR1 $15^{\mathrm{h}}$ & & $98250723^{9}$ & 98251294 & $6.2 \mathrm{E}-07$ & $2(1)$ & 98249859 & & $6.2 \mathrm{E}-04$ & $4(2)$ & 1.0E-07 \\
\hline & ZBTB38 & & 141086820 & 141087363 & 0.006 & $6(4)$ & & & 0.005 & & 0.005 \\
\hline & $L P P^{h}$ & & 187870621 & 187871538 & $1.5 \mathrm{E}-05$ & $11(5)$ & & & 0.001 & & $1.1 \mathrm{E}-04$ \\
\hline & C3orf43 & 21,882 & 196255632 & 196256223 & 9.7E-04 & $5(3)$ & & & 0.004 & & $1.8 \mathrm{E}-04$ \\
\hline \multirow[t]{5}{*}{4} & PCGF3 & & 737005 & 738199 & 0.002 & $8(2)$ & 736328 & & 0.001 & $12(4)$ & $2.5 \mathrm{E}-05$ \\
\hline & FGFRL1 & -1776 & 1003208 & 1003834 & $1.5 \mathrm{E}-04$ & $3(2)$ & & & 0.002 & & $2.0 \mathrm{E}-04$ \\
\hline & PRDM8 & & $81117647^{9}$ & 81119473 & $6.7 E-13$ & $11(10)$ & & & $2.9 \mathrm{E}-13$ & & $6.7 E-06$ \\
\hline & NHEDC1 & & 103940711 & 103941300 & $6.8 \mathrm{E}-14$ & $11(10)$ & & & 2.7E-10 & & $6.2 \mathrm{E}-05$ \\
\hline & CFI & & 110724358 & 110724834 & 0.006 & $2(2)$ & & & 0.009 & & $4.4 \mathrm{E}-04$ \\
\hline \multirow[t]{6}{*}{5} & $A H R R^{h}$ & & $373378^{9}$ & 374425 & 4.6E-17 & $5(2)$ & & 373887 & $4.8 \mathrm{E}-05$ & $2(1)$ & $6.5 \mathrm{E}-13$ \\
\hline & & & $392920^{9}$ & 393366 & $5.8 \mathrm{E}-08$ & $3(3)$ & & & $3.9 \mathrm{E}-08$ & & 4.7E-06 \\
\hline & LPCAT1 & & 1494980 & 1495356 & 0.001 & $5(4)$ & & & 0.003 & & 0.001 \\
\hline & LINC01019 & $-236,319$ & 3180918 & 3180947 & 0.006 & $2(2)$ & & 3182108 & $6.0 \mathrm{E}-04$ & $5(4)$ & 5.7E-04 \\
\hline & FLJ44606 & & 126408756 & 126409553 & $7.0 \mathrm{E}-07$ & $13(11)$ & & & $1.9 \mathrm{E}-06$ & & 0.001 \\
\hline & ADAMTS2 & & 178548229 & 178548700 & 0.002 & $3(3)$ & & & 0.003 & & $8.5 E-04$ \\
\hline \multirow[t]{9}{*}{6} & IER $^{\text {h }}$ & 9104 & 30720080 & 30720491 & $1.2 \mathrm{E}-06$ & $8(4)$ & & & 0.002 & & 1.7E-05 \\
\hline & LY6G6E & & 31683051 & 31683352 & $5.4 \mathrm{E}-05$ & $6(5)$ & & & $1.2 \mathrm{E}-04$ & & 0.002 \\
\hline & HLA-DPB1 & & 33047944 & 33049505 & $2.5 \mathrm{E}-09$ & $20(15)$ & & & $4.8 \mathrm{E}-08$ & & 0.002 \\
\hline & SYNGAPI ${ }^{\mathrm{h}}$ & & 33400477 & 33401542 & $6.9 \mathrm{E}-06$ & $9(7)$ & 33400021 & & $2.2 \mathrm{E}-05$ & $10(7)$ & 2.7E-04 \\
\hline & CRISP2 & & 49681178 & 49681774 & $5.5 \mathrm{E}-06$ & $9(8)$ & & & $5.5 \mathrm{E}-06$ & & $1.8 \mathrm{E}-04$ \\
\hline & UTRN & -4373 & 144607399 & 144608500 & 0.004 & $7(4)$ & 144607074 & & 0.010 & $8(4)$ & $2.6 \mathrm{E}-04$ \\
\hline & ZC $3 H 12 D^{h}$ & & 149805995 & 149806732 & $2.3 \mathrm{E}-15$ & $10(10)$ & & & $1.9 \mathrm{E}-14$ & & 8.7E-05 \\
\hline & TIAM $2^{h}$ & & 155537595 & 155538155 & $1.6 \mathrm{E}-05$ & $8(5)$ & & & 3.7E-05 & & $7.6 \mathrm{E}-04$ \\
\hline & THBS2 & & 169653612 & 169654719 & $9.5 \mathrm{E}-04$ & $11(4)$ & & 169654842 & $7.0 \mathrm{E}-04$ & $12(4)$ & $5.3 \mathrm{E}-04$ \\
\hline \multirow[t]{3}{*}{7} & GNA12 ${ }^{\mathrm{h}}$ & & 2768988 & 2770410 & 4.7E-06 & $5(5)$ & 2769253 & & $7.4 \mathrm{E}-05$ & $4(4)$ & $3.0 \mathrm{E}-05$ \\
\hline & TRG-ASI $1^{\mathrm{h}}$ & $-29,710$ & 38350464 & 38351468 & $2.0 \mathrm{E}-06$ & $7(6)$ & & & $1.1 \mathrm{E}-05$ & & 1.7E-04 \\
\hline & MYOIG $^{\mathrm{h}}$ & & $45001765^{g}$ & 45002919 & $5.5 E-14$ & $6(5)$ & & & 5.7E-09 & & $2.7 \mathrm{E}-06$ \\
\hline
\end{tabular}


Table 3 Differentially methylated regions in blood DNA in relation to current smoking compared to never smoking (multiple-testing corrected $p<0.01$ at DMRcate and comb-p, ordered by chromosomal location) (Continued)

\begin{tabular}{|c|c|c|c|c|c|c|c|c|c|c|c|}
\hline & INSIGI & 61,195 & 155150681 & 155151427 & 0.007 & $4(3)$ & & & 0.002 & & 0.003 \\
\hline \multirow[t]{4}{*}{8} & DEFA4 & & 6795162 & 6796618 & 2.0E-04 & $4(4)$ & 6794872 & & 1.7E-05 & $5(4)$ & 4.0E-05 \\
\hline & $E P B 49^{h}$ & & 21915184 & 21915510 & 0.004 & $2(2)$ & 21914287 & 21916853 & $5.3 \mathrm{E}-05$ & $11(6)$ & 0.002 \\
\hline & TRAPPC9 & & 141057285 & 141057827 & 3.7E-06 & $5(5)$ & & & $2.1 \mathrm{E}-06$ & & 2.0E-04 \\
\hline & GL/4 & & 144358043 & 144359316 & 0.001 & $5(5)$ & & & $1.5 E-05$ & & 0.002 \\
\hline \multirow[t]{2}{*}{9} & CD72 & & 35609853 & 35610380 & 0.002 & $2(2)$ & & & 0.007 & & 1.1 $\mathrm{E}-04$ \\
\hline & $C I Z 1$ & & 130955135 & 130956057 & 0.001 & $4(3)$ & & 130955436 & 0.004 & $3(3)$ & 0.001 \\
\hline \multirow[t]{5}{*}{10} & SNCG & & 88717926 & 88718393 & $5.5 \mathrm{E}-04$ & $5(5)$ & & & $3.8 \mathrm{E}-04$ & & 0.003 \\
\hline & $\operatorname{SLC16A12^{\mathrm {h}}}$ & & 91296252 & 91296457 & $1.6 \mathrm{E}-04$ & 3(3) & & & 0.004 & & 4.4E-04 \\
\hline & $L G / 1$ & & 95517382 & 95517895 & $6.3 \mathrm{E}-04$ & $7(4)$ & & & 0.002 & & 5.6E-04 \\
\hline & $N K \times 2-3$ & -4844 & $101287381^{9}$ & 101287846 & $8.2 \mathrm{E}-06$ & $5(3)$ & & & 1.3E-04 & & 7.4E-06 \\
\hline & GRK5 & & 121171859 & 121172898 & $6.4 \mathrm{E}-04$ & $5(4)$ & & & $2.4 \mathrm{E}-04$ & & 4.1E-04 \\
\hline \multirow[t]{6}{*}{11} & C11orf2 $1^{\mathrm{h}}$ & & 2321770 & 2322674 & $1.2 \mathrm{E}-05$ & $18(7)$ & & 2323938 & $1.3 \mathrm{E}-04$ & $33(8)$ & $5.9 \mathrm{E}-04$ \\
\hline & C11orf41 & & 33562503 & 33563377 & 7.0E-04 & $4(4)$ & & 33563946 & $2.3 \mathrm{E}-04$ & $5(4)$ & $5.4 \mathrm{E}-04$ \\
\hline & $N E A T 7^{\mathrm{h}}$ & 4664 & 65194933 & 65196227 & $2.2 \mathrm{E}-05$ & $7(7)$ & & 65196696 & $3.0 \mathrm{E}-05$ & $10(7)$ & 4.9E-04 \\
\hline & $A C Y 3$ & & 67418045 & 67418405 & $1.1 \mathrm{E}-09$ & $12(11)$ & & & 8.7E-08 & & 1.3E-04 \\
\hline & CCND1 & & $69462660^{9}$ & 69463323 & $2.4 \mathrm{E}-06$ & $6(3)$ & & & 1.7E-04 & & $7.5 \mathrm{E}-07$ \\
\hline & $A M I C A I^{h}$ & & 118084920 & 118085736 & 0.005 & $4(4)$ & & & 0.002 & & 0.003 \\
\hline \multirow[t]{6}{*}{12} & IFFO1 & & 6657744 & 6658945 & 2.7E-04 & $10(5)$ & & 6659524 & $2.2 \mathrm{E}-04$ & $12(5)$ & $8.1 \mathrm{E}-05$ \\
\hline & $M G P$ & & 15038440 & 15039432 & $9.5 \mathrm{E}-04$ & 4(3) & & & $3.5 \mathrm{E}-05$ & & $9.3 \mathrm{E}-05$ \\
\hline & KRT7 & & 52638005 & 52638592 & 0.002 & $3(2)$ & & & 0.005 & & $1.5 \mathrm{E}-04$ \\
\hline & ZNF385A & & 54778312 & 54779175 & 0.002 & $4(3)$ & & & 0.008 & & 0.001 \\
\hline & RP11-474D1.3 & 36,620 & 130554977 & 130555091 & $1.8 \mathrm{E}-04$ & $3(3)$ & & & $9.4 \mathrm{E}-04$ & & 1.7E-04 \\
\hline & STX2 & $-73,033$ & 131199848 & 131201112 & $7.2 \mathrm{E}-04$ & $10(4)$ & 131198873 & 131201268 & 0.008 & $12(5)$ & $6.5 \mathrm{E}-05$ \\
\hline \multirow[t]{3}{*}{14} & $L G M N$ & & 93170710 & 93170970 & 0.002 & $3(3)$ & & & 0.008 & & $6.6 \mathrm{E}-05$ \\
\hline & $E V L$ & & 100610071 & 100610667 & $9.8 \mathrm{E}-05$ & $6(4)$ & & & $1.9 \mathrm{E}-04$ & & 4.0E-04 \\
\hline & RIN3 & & 92981121 & 92981666 & 1.6E-05 & $3(3)$ & & & $2.1 \mathrm{E}-05$ & & $1.8 \mathrm{E}-04$ \\
\hline 15 & CALML4 & & $68498251^{9}$ & 68499367 & $2.6 \mathrm{E}-06$ & $5(2)$ & 68497992 & & 0.002 & $6(2)$ & $2.9 \mathrm{E}-07$ \\
\hline \multirow[t]{2}{*}{16} & PRR25 & & 854168 & 854640 & 0.002 & $4(3)$ & & 855449 & 0.002 & $6(4)$ & 7.7E-04 \\
\hline & $B C L 7 C$ & & 30906810 & 30907246 & 0.001 & $2(2)$ & & 30907560 & $8.0 E-04$ & $3(3)$ & $9.0 \mathrm{E}-04$ \\
\hline \multirow[t]{7}{*}{17} & ALOX15B & & 7942137 & 7942743 & $1.1 \mathrm{E}-04$ & $6(5)$ & & & $2.4 \mathrm{E}-04$ & & $3.9 \mathrm{E}-04$ \\
\hline & NTN1 & & 9018806 & 9019336 & $2.0 \mathrm{E}-05$ & $5(4)$ & & & $5.5 \mathrm{E}-04$ & & $5.3 \mathrm{E}-04$ \\
\hline & SLFN12L & $-13,916$ & 33787402 & 33788026 & 0.003 & $4(4)$ & & & 0.001 & & $8.8 \mathrm{E}-04$ \\
\hline & CYB561 & & 61511069 & 61511829 & $4.9 \mathrm{E}-04$ & $4(4)$ & & & $9.3 \mathrm{E}-05$ & & $5.2 \mathrm{E}-04$ \\
\hline & CCDC57 & & 80076338 & 80076378 & $1.1 \mathrm{E}-04$ & $2(2)$ & & & 0.002 & & $2.2 \mathrm{E}-05$ \\
\hline & FOXK2 & & $80545020^{9}$ & 80545869 & 8.1E-08 & 11(6) & & & $2.6 \mathrm{E}-04$ & & $5.5 \mathrm{E}-06$ \\
\hline & $T B C D$ & & 80870107 & 80870923 & 0.001 & $5(3)$ & & 80871405 & 0.002 & 7(4) & $1.8 \mathrm{E}-04$ \\
\hline 18 & C18orf1 & & 13611370 & 13611824 & 0.007 & $6(4)$ & & & 0.009 & & 0.003 \\
\hline \multirow[t]{3}{*}{19} & GNG7 & & 2543602 & 2544100 & 0.008 & $5(2)$ & 2542837 & & 0.002 & $6(3)$ & $6.4 \mathrm{E}-04$ \\
\hline & MAN2B1 & & 12758416 & 12759546 & 0.004 & $7(4)$ & & & 0.001 & & 0.002 \\
\hline & LAIR1 & & 54876446 & 54876795 & $1.8 \mathrm{E}-04$ & $5(4)$ & & & $8.1 \mathrm{E}-04$ & & 2.3E-04 \\
\hline 20 & C20orf27 & & 3745817 & 3746315 & 0.002 & $2(2)$ & & & 0.004 & & $8.8 \mathrm{E}-05$ \\
\hline
\end{tabular}


Table 3 Differentially methylated regions in blood DNA in relation to current smoking compared to never smoking (multiple-testing corrected $p<0.01$ at DMRcate and comb-p, ordered by chromosomal location) (Continued)

\begin{tabular}{|c|c|c|c|c|c|c|c|c|}
\hline \multirow[t]{3}{*}{22} & SYNGR1 & & $39759864^{9}$ & 39760267 & $1.2 \mathrm{E}-07$ & $5(5)$ & $1.2 \mathrm{E}-06$ & $2.5 \mathrm{E}-06$ \\
\hline & SHISAB & -978 & 42304331 & 42304580 & 1.4E-04 & $2(2)$ & $6.9 \mathrm{E}-04$ & $2.3 \mathrm{E}-05$ \\
\hline & ODF3B & & 50970943 & 50971140 & 4.2E-04 & 3(3) & 0.002 & $1.6 \mathrm{E}-04$ \\
\hline
\end{tabular}

Empty cells in "Start," "End," and "\#CpGs" for comb-p represent the same regional information compare to results in DMRcate. DMRs ordered by $\mathrm{p}$ values can be found in Additional file 11

${ }^{\mathrm{a} C h r o m o s o m e}$

${ }^{\mathrm{b}}$ Minimum distance to transcription start site of the mapped gene (basepair)

'Physical position (basepair, National Center for Biotechnology Information human reference genome assembly Build 37.3)

${ }^{\mathrm{d}}$ False discovery rate

${ }^{\mathrm{e}}$ Number of probes in the region (number of $\mathrm{CpGs}$ of nominal statistical significance)

${ }^{\mathrm{f}} \mathrm{P}$ of Sidak multiple-testing correction

${ }^{9}$ Region including significant (FDR <0.05) differentially methylated probes from our epigenome-wide association study (EWAS)

${ }^{\mathrm{h}}$ Gene identified in previous EWASs of smoking

'Minimum $p$ values among unadjusted $p$ values of $C p G$ s in each region

Table 4 CpGs differentially methylated in relation to smoking status also related to quantitative measures of smoking $(p$ correlation $<0.05$, ordered by chromosomal location)

\begin{tabular}{|c|c|c|c|c|c|c|c|}
\hline \multirow[t]{2}{*}{$\overline{C h r^{a}}$} & \multirow[t]{2}{*}{ Gene } & \multirow{2}{*}{$\begin{array}{l}\text { Distance } \\
\text { to gene }\end{array}$} & \multirow[t]{2}{*}{ Probe } & \multicolumn{2}{|c|}{ Epigenome-wide association study } & \multirow[t]{2}{*}{$\rho^{d}$} & \multirow[t]{2}{*}{$P_{\rho}$} \\
\hline & & & & $\overline{\text { Coef }^{c}}$ & $p$ & & \\
\hline \multicolumn{8}{|c|}{ Urine cotinine in current smokers $(N=31)$} \\
\hline 1 & GNG12 & & $\operatorname{cg} 25189904^{e, f}$ & -0.134 & $1.4 \mathrm{E}-06$ & -0.40 & 0.027 \\
\hline 3 & GPR15 & & $\operatorname{cg} 19859270^{e, f}$ & -0.027 & 1.0E-07 & -0.56 & 0.001 \\
\hline 4 & MTNR1A & & cg22261866 & -0.063 & $1.6 \mathrm{E}-06$ & 0.37 & 0.041 \\
\hline 5 & AHRR & & $\mathrm{cg} 05575921^{\mathrm{e}, \mathrm{f}}$ & -0.203 & $6.5 \mathrm{E}-13$ & -0.43 & 0.016 \\
\hline 15 & FAM82A2 & & $\operatorname{cg} 19440278$ & 0.007 & 7.0E-06 & -0.43 & 0.016 \\
\hline 19 & $F 2 R L 3$ & & $\mathrm{cg} 03636183^{\mathrm{e}, \mathrm{f}}$ & -0.128 & $2.0 \mathrm{E}-08$ & -0.56 & 0.001 \\
\hline \multicolumn{8}{|c|}{ Pack-year in current smokers $(N=31)$} \\
\hline 1 & NT5C1A & & cg00990022 & -0.04 & $5.5 \mathrm{E}-06$ & 0.39 & 0.036 \\
\hline 6 & ZBTB9 & & cg03945003 & -0.023 & $3.9 \mathrm{E}-06$ & 0.40 & 0.031 \\
\hline 10 & JAKMIP3 & & $\operatorname{cg} 19134728^{e}$ & -0.023 & $1.2 \mathrm{E}-05$ & 0.37 & 0.045 \\
\hline 11 & $H P X$ & & $\operatorname{cg} 25426350$ & -0.03 & $2.5 \mathrm{E}-06$ & 0.44 & 0.016 \\
\hline 11 & CCND1 & & cg09520904 & -0.036 & $7.5 \mathrm{E}-07$ & -0.44 & 0.015 \\
\hline 21 & RNF160 & & $\operatorname{cg} 13662262$ & -0.01 & $9.2 \mathrm{E}-06$ & 0.44 & 0.015 \\
\hline \multicolumn{8}{|c|}{ Time since quit smoking in former smokers $(N=30)$} \\
\hline 1 & $|F| 16$ & -9970 & cg19707735 & -0.035 & 1.0E-04 & 0.47 & 0.009 \\
\hline 2 & CLASP1 & & cg22346073 & -0.052 & 8.0E-07 & 0.43 & 0.017 \\
\hline 3 & ARHGEF3 & & $\operatorname{cg} 25799109^{e}$ & -0.076 & 4.4E-05 & -0.44 & 0.016 \\
\hline 3 & KTELC1 & & cg16958524 & -0.029 & $6.9 \mathrm{E}-06$ & 0.39 & 0.033 \\
\hline 5 & SPEF2 & & cg08534016 & -0.050 & 0.001 & 0.42 & 0.021 \\
\hline 6 & ACOT13 & 16438 & cg09447457 & -0.010 & $1.2 \mathrm{E}-05$ & 0.39 & 0.034 \\
\hline 9 & BSPRY & & cg02003202 & -0.049 & $9.5 \mathrm{E}-06$ & 0.44 & 0.015 \\
\hline 15 & FAM82A2 & & cg21580007 & -0.049 & $9.0 \mathrm{E}-04$ & 0.47 & 0.009 \\
\hline
\end{tabular}

Results for current and former smokers showed regression coefficients and $p$ values from EWAS for current and former smokers, respectively ${ }^{a}$ Chromosome

${ }^{b}$ Distance to transcription start site of the mapped gene (basepair, based on National Center for Biotechnology Information human reference genome assembly Build 37.3)

'Regression coefficient from statistical model

${ }^{\mathrm{d}}$ Spearman correlation (rho) was used for urine cotinine and pack-years in current smokers and time since quit smoking in former smokers. The methylation values were adjusted for age, sex, body mass index, chronic obstructive pulmonary disease status, and estimated cell composition

eprobe identified in previous epigenome-wide association studies (EWASs) of smoking

fProbe identified in one previous EWAS of serum cotinine 
Table 5 Differential methylation in relation to current smoking for genes with transcripts differently expressed $(p<0.05)$ in relation to smoking pack-years (ordered by chromosomal location)

\begin{tabular}{|c|c|c|c|c|c|c|c|c|c|}
\hline \multirow{2}{*}{\multicolumn{2}{|c|}{$\begin{array}{l}\text { Differentially methylated probe } \\
\text { smoking (the Korean COPD col } \\
\text { Differentially methylated probe }\end{array}$}} & $\begin{array}{l}\text { in relati } \\
\text { ort) }\end{array}$ & on to cur & tt smoking compared & never & $\begin{array}{l}\text { Gene (distance to } \\
\text { gene }^{c} \text { ) }\end{array}$ & \multicolumn{3}{|c|}{$\begin{array}{l}\text { Differentially expressed genes } \\
\text { in relation to pack-years in lung } \\
\text { tissue (Asan Biobank) }\end{array}$} \\
\hline & & & & & & & & & \\
\hline $\mathrm{Chr}^{\mathrm{a}}$ & Probe & Coef $^{b}$ & $P$ & Genomic features & CpG island & & Transcript & Coef & $P$ \\
\hline 1 & cg20388635 & -0.013 & $1.3 \mathrm{E}-05$ & TSS200, promoter & Island & YTHDF2 & NM_001173128 & -0.019 & 0.047 \\
\hline \multirow[t]{3}{*}{2} & cg22346073 & -0.056 & $5.1 \mathrm{E}-08$ & 5'UTR & Shelf & CLASP1 & NM_015282 & 0.019 & $7.2 \mathrm{E}-04$ \\
\hline & cg19394739 & -0.012 & $3.5 \mathrm{E}-07$ & Body, promoter & Shore & DGUOK & NM_080916 & -0.079 & 0.003 \\
\hline & cg09059267 & -0.099 & $4.2 \mathrm{E}-06$ & & Island & DNPEP (-15098) & NM_012100 & -0.021 & 0.037 \\
\hline \multirow[t]{2}{*}{3} & cg01870865 & -0.045 & 1.0E-05 & TSS200, promoter & & TREX1 & NM_033629 & -0.018 & 0.023 \\
\hline & $\operatorname{cg} 19859270^{d}$ & -0.027 & $1.0 \mathrm{E}-07$ & 1st exon & & GPR15 & NM_005290 & 0.013 & $3.0 \mathrm{E}-04$ \\
\hline \multirow[t]{3}{*}{5} & $\operatorname{cg} 05575921^{d}$ & -0.203 & $6.5 \mathrm{E}-13$ & Body, enhancer & Shore & $A H R R^{\mathrm{e}}$ & NM_001242412 & 0.004 & 0.047 \\
\hline & $\operatorname{cg} 14817490^{d}$ & -0.078 & 4.7E-06 & Body, promoter, DHS & & & & & \\
\hline & $\operatorname{cg} 25648203^{d}$ & -0.079 & $6.2 \mathrm{E}-07$ & Body, enhancer, DHS & & & & & \\
\hline 6 & $\operatorname{cg} 23164938$ & -0.016 & $9.5 \mathrm{E}-06$ & TSS1500 & Shore & ESR1 & NM_000125 & 0.005 & 0.012 \\
\hline \multirow[t]{2}{*}{7} & cg05383910 & -0.042 & $2.1 \mathrm{E}-06$ & $5^{\prime} \cup T R$, enhancer & & $E L M O 1^{\mathrm{e}}$ & NR_038121 & 0.017 & 0.031 \\
\hline & cg20663219 & -0.054 & $9.4 \mathrm{E}-06$ & Body, DHS & Shelf & STXIA & NM_001165903 & -0.003 & 0.045 \\
\hline 10 & cg20723792 & -0.097 & $4.8 \mathrm{E}-10$ & Body, enhancer, DHS & & FAM53B & NM_014661 & 0.009 & 0.042 \\
\hline 11 & $\operatorname{cg} 25426350$ & -0.030 & $2.5 \mathrm{E}-06$ & TSS200 & & $H P X$ & NM_000613 & -0.003 & 0.024 \\
\hline 13 & cg17058676 & -0.028 & $2.5 \mathrm{E}-06$ & Body & Shore & CENPJ & NM_018451 & 0.003 & 0.035 \\
\hline 14 & cg16579351 & -0.017 & $1.2 \mathrm{E}-05$ & Body & & BRF1 & NM_001242788 & -0.012 & 0.041 \\
\hline 17 & cg13521620 & -0.052 & $1.2 \mathrm{E}-05$ & $5^{\prime} U T R$ & Shore & YPEL2 & NM_001005404 & 0.019 & 0.023 \\
\hline \multicolumn{10}{|c|}{ Differentially methylated region } \\
\hline Chr & Region & $\# C p G s$ & FDR & Genomic features & CpG island & & & & \\
\hline 1 & $230415343-230416101$ & 6 & 0.002 & 3'UTR & $\begin{array}{l}\text { Island, } \\
\text { shore }\end{array}$ & GALNT2 & NM_004481 & 0.033 & 0.018 \\
\hline 2 & 241975756-241976244 & 4 & $1.9 \mathrm{E}-06$ & Body, promoter, DHS & island & $S N E D 1^{\mathrm{e}}$ & NM_001080437 & 0.017 & 0.014 \\
\hline 3 & $98250723-98251294$ & 2 & $6.2 \mathrm{E}-07$ & TSS200, 1st exon & & GPR15 & NM_005290 & 0.013 & $3.0 \mathrm{E}-04$ \\
\hline 3 & $187870621-187871538$ & 11 & $1.5 \mathrm{E}-05$ & TSS1500, TSS200 & $\begin{array}{l}\text { Shore, } \\
\text { island }\end{array}$ & $L P p^{e}$ & NM_005578 & 0.019 & 0.018 \\
\hline 5 & $373378-374425$ & 5 & 4.6E-17 & Body, enhancer & $\begin{array}{l}\text { Shore, } \\
\text { island }\end{array}$ & $A H R R^{\mathrm{e}}$ & NM_001242412 & 0.004 & 0.047 \\
\hline 5 & 392920-393366 & 3 & $5.8 \mathrm{E}-08$ & Body, promoter, DHS & & & & & \\
\hline 7 & $2768988-2770410$ & 5 & 4.7E-06 & 3'UTR, enhancer & & GNA12 ${ }^{\mathrm{e}}$ & NM_007353 & 0.022 & 0.019 \\
\hline 17 & $61511069-61511829$ & 4 & $4.9 \mathrm{E}-04$ & $\begin{array}{l}\text { 3'UTR, body, } \\
\text { enhancer }\end{array}$ & $\begin{array}{l}\text { Shore, } \\
\text { island }\end{array}$ & CYB561 & NM_001017916 & -0.056 & 0.005 \\
\hline
\end{tabular}

Genomic features were based on Illumina's Annotation file and those for DMRs were based on CpGs at start and end position of each region. Categories for the features includes (1) Body, gene body; (2) 5'UTR, 5 prime untranslated region; (3) 3'UTR, 3 prime untranslated region; (4) TSS200, 200 basepair within transcription start site; (5) TSS1500, 1500 basepair within transcription start site; and (6) DHS, DNase I hypersensitivity site

${ }^{\mathrm{a}}$ Chromosome

${ }^{\mathrm{b}}$ Regression coefficient from statistical model

'Distance to transcription start site of the mapped gene (basepair, National Center for Biotechnology Information human reference genome assembly Build 37.3)

${ }^{\mathrm{d}}$ Probe identified in previous epigenome-wide association studies (EWASs) of smoking

${ }^{\mathrm{e}} \mathrm{Gene}$ identified in previous EWASs of smoking

Our replication look-up confirmed 70 DMPs in the same direction of methylation changes from previous EWASs at strict look-up level significance. Of these, 51 were replicated in one EWAS [26] from a Chinese population. Nineteen were never replicated in an East Asian population. We could not replicate the novel findings identified from the EWAS in Chinese [26].

We had only one female current smoker and six male never smokers. Because of this imbalance, our adjustment for gender may not eliminate potential bias in the 
Table 6 Look-up in the Korean COPD cohort of CpGs reported at least two epigenome-wide association studies (70 CpGs at FDR ${ }^{9}<0.05$, ordered by chromosomal location)

\begin{tabular}{|c|c|c|c|c|c|c|}
\hline $\mathrm{Chr}^{\mathrm{a}}$ & Gene & $\begin{array}{l}\text { Distance } \\
\text { to gene }\end{array}$ & Probe & Coef $^{c}$ & $p^{d}$ & References $^{\mathrm{e}}$ \\
\hline \multirow[t]{8}{*}{1} & GNG12 & & $\operatorname{cg} 25189904^{f}$ & -0.134 & $1.4 \mathrm{E}-06$ & $\begin{array}{l}\text { Guida et al. } 2015 \text { [12]; Besingi and Johansson 2014 [14]; Elliott et al. } 2014 \text { [16]; } \\
\text { Zeilinger et al. } 2013 \text { [22]; Tsaprouni et al. 2014[18]; Zhu et al. } 2016 \text { [26]. }\end{array}$ \\
\hline & & & $\operatorname{cg} 26764244$ & -0.055 & 0.010 & Guida et al. 2015 [12]; Harlid et al. 2014[17]. \\
\hline & GFI1 & & $\operatorname{cg} 12876356^{f}$ & -0.049 & $8.9 \mathrm{E}-04$ & $\begin{array}{l}\text { Guida et al. } 2015 \text { [12]; Besingi and Johansson } 2014 \text { [14]; Dogan et al. 2014[15]; } \\
\text { Zeilinger et al. } 2013 \text { [22]; Zhu et al. } 2016 \text { [26]. }\end{array}$ \\
\hline & & & $\operatorname{cg} 18316974$ & -0.014 & 0.006 & Guida et al. 2015 [12]; Besingi and Johansson 2014 [14]; Zeilinger et al. 2013 [22]. \\
\hline & & & cg09935388 & -0.106 & $8.1 \mathrm{E}-05$ & $\begin{array}{l}\text { Guida et al. } 2015 \text { [12]; Besingi and Johansson } 2014 \text { [14]; Dogan et al. 2014[15]; } \\
\text { Elliott et al. } 2014 \text { [16]; Zeilinger et al. } 2013 \text { [22]; Zhu et al. } 2016 \text { [26]. }\end{array}$ \\
\hline & $A V P R 1 B$ & & $\operatorname{cg} 08709672^{f}$ & -0.058 & $1.1 \mathrm{E}-06$ & $\begin{array}{l}\text { Guida et al. } 2015 \text { [12]; Besingi and Johansson } 2014 \text { [14]; Elliott et al. } 2014 \text { [16]; } \\
\text { Zeilinger et al. } 2013 \text { [22]; Zhu et al. } 2016 \text { [26]. }\end{array}$ \\
\hline & & & cg20295214 & -0.068 & $3.5 \mathrm{E}-04$ & $\begin{array}{l}\text { Guida et al. } 2015 \text { [12]; Besingi and Johansson } 2014 \text { [14]; Tsaprouni et al. 2014[18]; } \\
\text { Zeilinger et al. } 2013 \text { [22]. }\end{array}$ \\
\hline & PSEN2 & -55213 & cg03547355 & -0.034 & 0.016 & Guida et al. 2015 [12]; Tsaprouni et al. 2014[18]; Zeilinger et al. 2013 [22]. \\
\hline \multirow[t]{10}{*}{2} & LINC00299 & 195809 & $\operatorname{cg} 23079012^{f}$ & -0.023 & $3.8 \mathrm{E}-04$ & $\begin{array}{l}\text { Besingi and Johansson } 2014 \text { [14]; Tsaprouni et al. 2014[18]; Zeilinger et al. } 2013 \\
\text { [22]; Zhu et al. } 2016 \text { [26]. }\end{array}$ \\
\hline & NFE2L2 & & $\operatorname{cg} 26271591^{f}$ & -0.061 & 8.3E-04 & Guida et al. 2015 [12]; Besingi and Johansson 2014 [14]; Zeilinger et al. 2013 [22]. \\
\hline & GPR55 & & $\operatorname{cg} 19827923$ & -0.022 & 0.012 & Guida et al. 2015 [12]; Zhu et al. 2016 [26]. \\
\hline & ALPP & & $\operatorname{cg} 23667432$ & -0.027 & 0.013 & Guida et al. 2015 [12]; Zeilinger et al. 2013 [22]. \\
\hline & ECELTP2 & -90 & $\operatorname{cg} 27241845$ & -0.081 & 4.1E-04 & $\begin{array}{l}\text { Guida et al. } 2015 \text { [12]; Besingi and Johansson } 2014 \text { [14]; Zeilinger et al. } 2013 \text { [22]; } \\
\text { Tsaprouni et al. 2014[18]. }\end{array}$ \\
\hline & ALPPL2 & 11777 & $\operatorname{cg} 03329539^{f}$ & -0.064 & $3.9 \mathrm{E}-05$ & $\begin{array}{l}\text { Guida et al. } 2015 \text { [12]; Besingi and Johansson } 2014 \text { [14]; Dogan et al. 2014[15]; } \\
\text { Elliott et al. } 2014 \text { [16]; Tsaprouni et al. 2014[18]; Zeilinger et al. } 2013 \text { [22]; Zhu et al. } \\
2016 \text { [26]. }\end{array}$ \\
\hline & & 12850 & $\operatorname{cg} 05951221^{f}$ & -0.088 & 8.4E-09 & $\begin{array}{l}\text { Allione et al. } 2015 \text { [11]; Guida et al. } 2015 \text { [12]; Besingi and Johansson } 2014 \text { [14]; } \\
\text { Dogan et al. 2014[15]; Harlid et al. 2014[17]; Elliott et al. } 2014 \text { [16]; Tsaprouni et al. } \\
\text { 2014[18]; Shenker et al. 2013[19]; Zeilinger et al. } 2013 \text { [22]; Zhu et al. } 2016 \text { [26]. }\end{array}$ \\
\hline & & 13382 & cg01940273 & -0.090 & $1.4 \mathrm{E}-06$ & $\begin{array}{l}\text { Allione et al. } 2015 \text { [1 1]; Guida et al. } 2015 \text { [12]; Besingi and Johansson } 2014 \text { [14]; } \\
\text { Dogan et al. 2014[15]; Elliott et al. } 2014 \text { [16]; Tsaprouni et al. 2014[18]; Shenker et al. } \\
\text { 2013[19]; Zeilinger et al. } 2013 \text { [22]; Zhu et al. } 2016 \text { [26]. }\end{array}$ \\
\hline & & 13737 & $\operatorname{cg} 13193840^{f}$ & -0.027 & $1.1 \mathrm{E}-04$ & $\begin{array}{l}\text { Guida et al. } 2015 \text { [12]; Tsaprouni et al. 2014[18]; Zeilinger et al. } 2013 \text { [22]; Zhu et al. } \\
2016 \text { [26]. }\end{array}$ \\
\hline & SNED1 & & $\operatorname{cg} 26718213$ & 0.091 & 0.005 & Guida et al. 2015 [12]; Besingi and Johansson 2014 [14]. \\
\hline \multirow[t]{3}{*}{3} & GPX1 & & $\operatorname{cg} 18642234$ & -0.042 & 0.005 & Guida et al. 2015 [12]; Zeilinger et al. 2013 [22]; Zhu et al. 2016 [26]. \\
\hline & GPR15 & & cg19859270 & -0.027 & 1.0E-07 & $\begin{array}{l}\text { Guida et al. } 2015 \text { [12]; Besingi and Johansson } 2014 \text { [14]; Dogan et al. 2014[15]; } \\
\text { Harlid et al. 2014[17]; Elliott et al. 2014 [16]; Tsaprouni et al. 2014[18]; Sun et al. } \\
\text { 2013[20]; Zeilinger et al. } 2013 \text { [22]; Wan et al. 2012[24]; Breitling et al. 2011[25]; } \\
\text { Zaghlool et al. 2015[13]; Zhu et al. } 2016 \text { [26]. }\end{array}$ \\
\hline & CPOX & & cg02657160 & -0.030 & $1.8 \mathrm{E}-04$ & $\begin{array}{l}\text { Guida et al. } 2015 \text { [12]; Besingi and Johansson } 2014 \text { [14]; Dogan et al. 2014[15]; } \\
\text { Harlid et al. 2014[17]; Tsaprouni et al. 2014[18]; Zeilinger et al. } 2013 \text { [22]. }\end{array}$ \\
\hline \multirow[t]{5}{*}{5} & AHRR & & $\operatorname{cg} 11554391$ & -0.043 & $2.5 \mathrm{E}-04$ & Guida et al. 2015 [12]; Zeilinger et al. 2013 [22]; Zhu et al. 2016 [26]. \\
\hline & & & $\operatorname{cg} 12806681^{f}$ & -0.015 & 0.009 & $\begin{array}{l}\text { Guida et al. } 2015 \text { [12]; Besingi and Johansson } 2014 \text { [14]; Dogan et al. 2014[15]; } \\
\text { Zeilinger et al. } 2013 \text { [22]; Zhu et al. } 2016 \text { [26]. }\end{array}$ \\
\hline & & & $\operatorname{cg} 23916896^{f}$ & -0.063 & 0.006 & $\begin{array}{l}\text { Guida et al. } 2015 \text { [12]; Dogan et al. 2014[15]; Zeilinger et al. } 2013 \text { [22]; Zhu et al. } \\
2016 \text { [26]. }\end{array}$ \\
\hline & & & $\mathrm{cg} 01899089^{f}$ & -0.054 & 0.003 & $\begin{array}{l}\text { Guida et al. } 2015 \text { [12]; Besingi and Johansson } 2014 \text { [14]; Dogan et al. 2014[15]; } \\
\text { Zeilinger et al. } 2013 \text { [22]. }\end{array}$ \\
\hline & & & $\operatorname{cg} 05575921^{f}$ & -0.203 & $6.5 \mathrm{E}-13$ & $\begin{array}{l}\text { Allione et al. } 2015 \text { [11]; Guida et al. } 2015 \text { [12]; Besingi and Johansson } 2014 \text { [14]; } \\
\text { Dogan et al. 2014[15]; Harlid et al. } 2014[17] ; \text { Elliott et al. } 2014 \text { [16]; Tsaprouni et al. } \\
\text { 2014[18]; Shenker et al. 2013[19]; Zeilinger et al. } 2013 \text { [22]; Zaghlool et al. 2015[13]; } \\
\text { Philibert et al. 2012[23]; Philibert et al. 2013[21]; Zhu et al. } 2016 \text { [26]. }\end{array}$ \\
\hline
\end{tabular}


Table 6 Look-up in the Korean COPD cohort of CpGs reported at least two epigenome-wide association studies (70 CpGs at FDR ${ }^{9}<0.05$, ordered by chromosomal location) (Continued)

\begin{tabular}{|c|c|c|c|c|c|c|}
\hline & & & $\operatorname{cg} 14817490$ & -0.078 & 4.7E-06 & $\begin{array}{l}\text { Guida et al. } 2015 \text { [12]; Besingi and Johansson 2014 [14]; Elliott et al. } 2014 \text { [16]; } \\
\text { Tsaprouni et al. 2014[18]; Zeilinger et al. } 2013 \text { [22]; Zaghlool et al. 2015[13]; Zhu } \\
\text { et al. } 2016 \text { [26]. }\end{array}$ \\
\hline & & & $\operatorname{cg} 17287155$ & -0.023 & $1.8 \mathrm{E}-04$ & Guida et al. 2015 [12]; Dogan et al. 2014[15]; Zhu et al. 2016 [26]. \\
\hline & & & cg04551776 & -0.038 & $1.3 \mathrm{E}-04$ & Guida et al. 2015 [12]; Elliott et al. 2014 [16]; Zhu et al. 2016 [26]. \\
\hline & & & cg25648203 & -0.079 & $6.2 \mathrm{E}-07$ & $\begin{array}{l}\text { Allione et al. } 2015 \text { [11]; Guida et al. } 2015 \text { [12]; Besingi and Johansson } 2014 \text { [14]; } \\
\text { Dogan et al. 2014[15]; Elliott et al. } 2014 \text { [16]; Zeilinger et al. } 2013 \text { [22]; Tsaprouni } \\
\text { et al. 2014[18]; Zhu et al. } 2016 \text { [26]. }\end{array}$ \\
\hline & & & cg24090911 & -0.039 & 0.009 & $\begin{array}{l}\text { Guida et al. } 2015 \text { [12]; Tsaprouni et al. 2014[18]; Zeilinger et al. } 2013 \text { [22]; Zhu et al. } \\
2016 \text { [26]. }\end{array}$ \\
\hline \multirow[t]{4}{*}{6} & IER3 & 9104 & $\operatorname{cg} 06126421$ & -0.101 & $2.6 \mathrm{E}-04$ & $\begin{array}{l}\text { Allione et al. } 2015 \text { [11]; Guida et al. } 2015 \text { [12]; Besingi and Johansson } 2014 \text { [14]; } \\
\text { Dogan et al. 2014[15]; Elliott et al. } 2014 \text { [16]; Tsaprouni et al. 2014[18]; Shenker } \\
\text { et al. 2013[19]; Zeilinger et al. } 2013 \text { [22]; Zhu et al. } 2016 \text { [26]. }\end{array}$ \\
\hline & & 9132 & $\operatorname{cg} 14753356^{f}$ & -0.062 & 1.7E-05 & $\begin{array}{l}\text { Guida et al. } 2015 \text { [12]; Besingi and Johansson } 2014 \text { [14]; Zeilinger et al. } 2013 \text { [22]; } \\
\text { Zhu et al. } 2016 \text { [26]. }\end{array}$ \\
\hline & & 9227 & $\operatorname{cg} 24859433$ & -0.037 & 0.003 & $\begin{array}{l}\text { Guida et al. } 2015 \text { [12]; Besingi and Johansson 2014 [14]; Dogan et al. 2014[15]; } \\
\text { Tsaprouni et al. 2014[18]; Zeilinger et al. } 2013 \text { [22]; Zhu et al. } 2016 \text { [26]. }\end{array}$ \\
\hline & & 9233 & cg15342087 & -0.030 & 0.009 & $\begin{array}{l}\text { Guida et al. } 2015 \text { [12]; Besingi and Johansson } 2014 \text { [14]; Tsaprouni et al. 2014[18]; } \\
\text { Zeilinger et al. } 2013 \text { [22]; Zhu et al. } 2016 \text { [26]. }\end{array}$ \\
\hline 7 & GNA12 & & $\operatorname{cg} 18446336$ & -0.074 & 0.011 & Guida et al. 2015 [12]; Zhu et al. 2016 [26]. \\
\hline & MYO1G & & cg19089201 & 0.056 & $3.5 \mathrm{E}-05$ & Zeilinger et al. 2013 [22]; Zhu et al. 2016 [26]. \\
\hline & & & $\operatorname{cg} 22132788$ & 0.092 & 2.7E-06 & $\begin{array}{l}\text { Guida et al. } 2015 \text { [12]; Besingi and Johansson 2014 [14]; Elliott et al. } 2014 \text { [16]; } \\
\text { Zeilinger et al. } 2013 \text { [22]; Philibert et al. 2012[23]; Philibert et al. 2013[21]; Zhu et al. } \\
2016 \text { [26]. }\end{array}$ \\
\hline & & & cg04180046 & 0.103 & $2.3 \mathrm{E}-05$ & Zeilinger et al. 2013 [22]; Zhu et al. 2016 [26]. \\
\hline & & & $\operatorname{cg} 12803068$ & 0.156 & $4.8 \mathrm{E}-06$ & $\begin{array}{l}\text { Allione et al. } 2015 \text { [11]; Guida et al. } 2015 \text { [12]; Besingi and Johansson } 2014 \text { [14]; } \\
\text { Elliott et al. 2014 [16]; Zeilinger et al. } 2013 \text { [22]; Philibert et al. 2012[23]; Philibert } \\
\text { et al. 2013[21]; Zhu et al. } 2016 \text { [26]. }\end{array}$ \\
\hline & CNTNAP2 & & $\operatorname{cg} 21322436$ & -0.026 & 0.016 & Guida et al. 2015 [12]; Zeilinger et al. 2013 [22]; Zhu et al. 2016 [26]. \\
\hline & & & $\operatorname{cg} 25949550$ & -0.026 & $5.2 \mathrm{E}-05$ & $\begin{array}{l}\text { Guida et al. } 2015 \text { [12]; Besingi and Johansson } 2014 \text { [14]; Zeilinger et al. } 2013 \text { [22]; } \\
\text { Zhu et al. } 2016 \text { [26]. }\end{array}$ \\
\hline 8 & MYST3 & & $\operatorname{cg} 14316231$ & -0.029 & 0.007 & Guida et al. 2015 [12]; Zhu et al. 2016 [26]. \\
\hline 9 & SLC44A1 & -1580 & $\operatorname{cg} 01692968^{f}$ & -0.038 & 0.004 & $\begin{array}{l}\text { Guida et al. } 2015 \text { [12]; Besingi and Johansson } 2014 \text { [14]; Zeilinger et al. } 2013 \text { [22]; } \\
\text { Zhu et al. } 2016 \text { [26]. }\end{array}$ \\
\hline 10 & ZMIZ1 & & $\operatorname{cg} 03450842^{f}$ & -0.041 & 0.004 & Guida et al. 2015 [12]; Besingi and Johansson 2014 [14]. \\
\hline \multirow[t]{9}{*}{11} & KCNQ10T1 & & cg01744331 & -0.030 & $8.4 \mathrm{E}-04$ & Guida et al. 2015 [12]; Zeilinger et al. 2013 [22]. \\
\hline & & & $\operatorname{cg} 07123182^{f}$ & -0.031 & $1.1 \mathrm{E}-05$ & $\begin{array}{l}\text { Guida et al. } 2015 \text { [12]; Besingi and Johansson } 2014 \text { [14]; Elliott et al. } 2014 \text { [16]; } \\
\text { Zeilinger et al. } 2013 \text { [22]; Zhu et al. } 2016 \text { [26]. }\end{array}$ \\
\hline & & & $\operatorname{cg} 16556677^{f}$ & -0.051 & $6.7 \mathrm{E}-04$ & Guida et al. 2015 [12]; Zeilinger et al. 2013 [22]; Zhu et al. 2016 [26]. \\
\hline & & & $\operatorname{cg} 26963277^{f}$ & -0.043 & $6.2 \mathrm{E}-04$ & $\begin{array}{l}\text { Guida et al. } 2015 \text { [12]; Besingi and Johansson } 2014 \text { [14]; Zeilinger et al. } 2013 \text { [22]; } \\
\text { Zhu et al. } 2016 \text { [26]. }\end{array}$ \\
\hline & LRP5 & & $\operatorname{cg} 21611682$ & -0.045 & 0.005 & $\begin{array}{l}\text { Guida et al. } 2015 \text { [12]; Besingi and Johansson } 2014 \text { [14]; Zeilinger et al. } 2013 \text { [22]; } \\
\text { Tsaprouni et al. 2014[18]; Zhu et al. } 2016 \text { [26]. }\end{array}$ \\
\hline & & & $\operatorname{cg} 10420527$ & -0.031 & 0.013 & Guida et al. 2015 [12]; Zhu et al. 2016 [26]. \\
\hline & & & $\operatorname{cg} 14624207^{f}$ & -0.040 & 0.002 & Guida et al. 2015 [12]; Zeilinger et al. 2013 [22]; Zhu et al. 2016 [26]. \\
\hline & ARRB1 & & cg01901332 & -0.057 & 0.008 & Guida et al. 2015 [12]; Besingi and Johansson 2014 [14]; Zeilinger et al. 2013 [22]. \\
\hline & PRSS23 & & cg23771366 & -0.062 & 0.002 & $\begin{array}{l}\text { Guida et al. } 2015 \text { [12]; Elliott et al. } 2014 \text { [16]; Zeilinger et al. } 2013 \text { [22]; Zhu et al. } \\
2016 \text { [26]. }\end{array}$ \\
\hline 12 & ETV6 & & $\operatorname{cg} 07986378^{f}$ & -0.069 & $3.6 \mathrm{E}-04$ & Guida et al. 2015 [12]; Besingi and Johansson 2014 [14]; Zhu et al. 2016 [26]. \\
\hline
\end{tabular}


Table 6 Look-up in the Korean COPD cohort of CpGs reported at least two epigenome-wide association studies $(70$ CpGs at FDR $<0.05$, ordered by chromosomal location) (Continued)

\begin{tabular}{|c|c|c|c|c|c|c|}
\hline \multirow[t]{2}{*}{14} & \multicolumn{2}{|l|}{ C14orf43 } & cg01731783 & -0.025 & 0.009 & $\begin{array}{l}\text { Guida et al. } 2015 \text { [12]; Dogan et al. 2014[15]; Elliott et al. } 2014 \text { [16]; Zeilinger et al. } \\
2013 \text { [22]. }\end{array}$ \\
\hline & \multicolumn{2}{|l|}{ ITPK1 } & $\operatorname{cg} 05284742$ & -0.055 & $2.5 \mathrm{E}-05$ & $\begin{array}{l}\text { Guida et al. } 2015 \text { [12]; Besingi and Johansson } 2014 \text { [14]; Zeilinger et al. } 2013 \text { [22]; } \\
\text { Zhu et al. } 2016 \text { [26]. }\end{array}$ \\
\hline \multirow[t]{2}{*}{15} & \multicolumn{2}{|l|}{ SEMATA } & cg00310412 & -0.036 & 0.008 & Guida et al. 2015 [12]; Besingi and Johansson 2014 [14]; Zeilinger et al. 2013 [22]. \\
\hline & \multicolumn{2}{|l|}{ ANPEP } & $\operatorname{cg} 23161492$ & -0.055 & 0.001 & $\begin{array}{l}\text { Guida et al. } 2015 \text { [12]; Besingi and Johansson } 2014 \text { [14]; Zeilinger et al. } 2013 \text { [22]; } \\
\text { Zhu et al. } 2016 \text { [26]. }\end{array}$ \\
\hline \multirow[t]{2}{*}{16} & \multicolumn{2}{|l|}{ XYLT1 } & $\operatorname{cg} 16794579^{f}$ & -0.039 & 0.004 & Guida et al. 2015 [12]; Besingi and Johansson 2014 [14]. \\
\hline & FBRS & -4029 & cg07069636 & -0.023 & 0.006 & Guida et al. 2015 [12]; Zhu et al. 2016 [26]. \\
\hline 17 & \multicolumn{2}{|l|}{ LOC100130933 } & $\operatorname{cg} 07251887^{f}$ & -0.070 & $2.4 \mathrm{E}-04$ & Guida et al. 2015 [12]; Zeilinger et al. 2013 [22]. \\
\hline \multirow[t]{5}{*}{19} & CIRBP & -1591 & cg00073090 & -0.031 & 0.002 & Guida et al. 2015 [12]; Zeilinger et al. 2013 [22]; Zhu et al. 2016 [26]. \\
\hline & \multicolumn{2}{|l|}{ MOBKLL2A } & cg15187398 & -0.048 & 0.013 & Guida et al. 2015 [12]; Zeilinger et al. 2013 [22]; Zhu et al. 2016 [26]. \\
\hline & MIR23A & 3767 & cg05339037 & -0.025 & 0.008 & Guida et al. 2015 [12]; Zhu et al. 2016 [26]. \\
\hline & \multicolumn{2}{|l|}{$F 2 R L 3$} & $\operatorname{cg} 03636183^{f}$ & -0.128 & 2.0E-08 & $\begin{array}{l}\text { Allione et al. } 2015 \text { [11]; Guida et al. } 2015 \text { [12]; Besingi and Johansson } 2014 \text { [14]; } \\
\text { Dogan et al. 2014[15]; Harlid et al. 2014[17]; Elliott et al. } 2014 \text { [16]; Tsaprouni et al. } \\
\text { 2014[18]; Shenker et al. 2013[19]; Sun et al. 2013[20]; Zeilinger et al. } 2013 \text { [22]; Wan } \\
\text { et al. 2012[24]; Breitling et al. } 2011 \text { [25]; Zaghlool et al. 2015[13]; Zhu et al. } 2016 \text { [26]. }\end{array}$ \\
\hline & \multicolumn{2}{|l|}{ PPP1R15A } & cg03707168 & -0.034 & 0.009 & Guida et al. 2015 [12]; Besingi and Johansson 2014 [14]. \\
\hline 20 & \multicolumn{2}{|l|}{ ATP9A } & cg07339236 & -0.039 & $1.0 \mathrm{E}-04$ & Guida et al. 2015 [12]; Zeilinger et al. 2013 [22]; Zhu et al. 2016 [26]. \\
\hline 21 & \multicolumn{2}{|l|}{ NCRNA00114 } & $\operatorname{cg} 06595162^{f}$ & -0.034 & 0.005 & Guida et al. 2015 [12]; Zeilinger et al. 2013 [22]. \\
\hline 22 & \multicolumn{2}{|l|}{ NCF4 } & cg02532700 & -0.049 & 0.003 & Guida et al. 2015 [12]; Zeilinger et al. 2013 [22]; Zhu et al. 2016 [26]. \\
\hline
\end{tabular}

${ }^{\mathrm{a} C h r o m o s o m e}$

${ }^{b}$ Distance to transcription start site of the mapped gene (basepair, National Center for Biotechnology Information human reference genome assembly Build 37.3) ${ }^{\mathrm{C}}$ Regression coefficient from statistical model

${ }^{\mathrm{d}}$ Statistical significance from statistical model

eArticles reporting CpGs as smoking-associated differential methylation sites at genome-wide level

fProbe differentially methylated in both current and former smokers compared to never smokers in our epigenome-wide association study

${ }^{9}$ Correction for 18 tests at the look-up

smoking results. We identified one EWAS of gender using Illumina's $450 \mathrm{k}$ array [13] in blood DNA $(n=123)$. In their supplementary table, they presented 274 genderassociated CpGs of genome-wide significance $(p<1.07 \mathrm{E}$ $-07)$ located in autosomes. None of our 108 smoking DMPs (FDR < 0.05) were among those suggesting that our top findings do not reflect the gender imbalance.

In our EWAS, we used COPD status as a covariate. The disease status could be a confounding factor. For 108 FDR-significant DMPs related to current smoking, we checked the association between COPD status and DNA methylation under two statistical models. Model 1 included covariates of age, sex, height, and estimated cell-type compositions; model 2 contained additional covariates of smoking status and pack-years. None of our DMPs were statistically significantly associated with COPD under either model (FDR $\leq 0.05$ after correcting for 108 tests). Sixteen CpGs were nominally related to COPD at uncorrected $p<0.05$ (Additional file 9: Table S9).

There are limitations and strengths in this study. First, these data were cross-sectional which limits causal inference regarding resolution of effects with cessation of smoking. Second, we do not have a replication dataset from an independent Korean, or similar, population.
Therefore, there is a chance of false positives among our novel findings. Third, the study population was drawn from a COPD cohort. Although we adjusted for the disease status in the regression models, the possibility of some type of selection bias could be raised. Fourth, we used blood DNA methylation to examine effects of smoking. The use of blood DNA methylation changes can be limited due to cell- and tissue-specific characteristics of methylation. However, our findings of differential methylation were adjusted for estimated cell-type proportions. We also confirmed differential transcriptome patterns in relation to pack-years in lung tissue at multiple loci.

Our study also has strengths. This is one of the few studies in Asian populations and the first in Koreans. We verified self-reported non-smoking status with urine cotinine values. Underreporting of smoking status in surveys occurs [61] and the nondifferential misclassification could distort association results. We also implemented two DMR approaches to provide significant DMRs in our EWAS. The methodologies for the discovery of DMRs have been developed and revised over several years, and it has been reported that the performance of DMRcate and comb-p were superior to those of others [44]. We were also able to examine whether genes with differential 
methylation in relation to smoking also showed differential transcription in relation to smoking in lung tissue, an important target for smoking related pathology.

\section{Conclusions}

Our study in Koreans, we discovered novel smokingassociated DNA methylation changes in blood and also confirmed many previous findings mostly identified in Caucasians. Observed correlations between methylation levels and quantitative measures of smoking exposures support the utility of blood DNA methylation biomarkers for smoking intensity and history. Our evaluation of differential gene expression profiles of corresponding genes in lung tissues supports the potential functional importance of our methylation findings.

\section{Additional files}

Additional file 1: Table S2. Relevant parameters for differential methylated region calling. (DOC $32 \mathrm{~kb}$ )

Additional file 2: Figure S2. Regional visualization of the association between current smoking and DNA methylation in blood. (DOC 555 kb)

Additional file 3: Table S1. Epigenome-wide association results of current smoking (compared to never smoking). (XLSX 30455 kb)

Additional file 4: Table S3. CpGs differentially methylated in blood DNA in relation to current smoking compared to never smoking: 108 probes (FDR $<0.05$, ordered by chromosomal location). (DOC $166 \mathrm{~kb}$ )

Additional file 5: Figure S1. Manhattan plot and quantile-quantile plot. (DOC 468 kb)

Additional file 6: Table S4. Table S4 CpGs included in the top five differentially methylated regions from each analysis: DMRcate and comb-p (ordered by software and chromosomal location). (DOC 146 kb)

Additional file 7: Table S5. CpGs differentially methylated in blood DNA in relation to current and former smoking compared to never smoking, 104 probes (FDR ${ }^{*}<0.05$, ordered by chromosomal location). (DOC $177 \mathrm{~kb}$ )

Additional file 8: Table S6. Enriched networks in genes related to current smoking. (DOC $35 \mathrm{~kb}$ )

Additional file 9: Table S9. Association results of COPD status and DNA methylation at the 16 CpGs with unadjusted $p<0.05$ in Model 1 or 2 . (DOC $47 \mathrm{~kb}$ )

Additional file 10: Table S7. Top 30 CpGs differentially methylated in blood DNA in relation to current smoking compared to never smoking (FDR $\leq 0.05$, ordered by $p$ values). (DOC $69 \mathrm{~kb}$ )

Additional file 11: Table S8. Differentially methylated regions in blood DNA in relation to current smoking compared to never smoking (multiple-testing corrected $p<0.01$ at DMRcate and comb-p, ordered by $p$ values). (DOC $173 \mathrm{~kb}$ )

\footnotetext{
Abbreviations

ACOT11: Acyl-CoA thioesterase 11; ACY3: Aminoacylase 3; ADAMTS2: ADAM metallopeptidase with thrombospondin type 1 motif 2; ADCYAP1R1: ADCYAP receptor type 1; AHDC1: AT-hook DNA binding motif containing 1; AHRR: Arylhydrocarbon receptor repressor; ALDOA: Aldolase, fructose-bisphosphate A; ALOX15B: Arachidonate 15-lipoxygenase, type B; ALPPL2: Alkaline phosphatase, placental-like 2; BCL7C: BCL tumor suppressor 7C; BMI: Body mass index: BMIQ: Beta MIxture Quantile dilation; bp: Basepair; C11 orf21: Chromosome 11 open reading frame 21; C20orf27: Chromosome 20 open reading frame 27; C5orf63 (clone name: FL44606): Chromosome 5 open reading frame 63; CALML4: Calmodulin-like 4; CASZ1: Castor zinc finger 1; CCDC57: Coiled-coil domain containing 57; CCND1: Cyclin D1; CD33: CD33 molecule; CD72: CD72
}

molecule; CDK2AP1: Cyclin-dependent kinase 2 associated protein 1; CFAP36 (alias CCDC104): Cilia and flagella associated protein 36; CFl: Complement factor 1; CFL2: Cofilin 2; CIZ1: CDKN1A interacting zinc finger protein 1; CLASP1: Cytoplasmic linker associated protein 1; COPD: Chronic obstructive pulmonary disease; CORO2B: Coronin 2B; CpG: Cytosine-phosphate-guanine; CRISP2: Cysteine rich secretory protein 2; CYB561: Cytochrome b561; DDA1: DET1 and DDB1 associated 1; DEFA4: Defensin alpha 4;

DGUOK: Deoxyguanosine kinase; DIXDC1: DIX domain containing 1; DMP: Differentially methylated probe; DMR: Differentially methylated region; E2F8: E2F transcription factor 8; EPB49: Dematin actin binding protein; EVL: Enah/Vasp-like; EWAS: Epigenome-wide association study; EXOC3L4: Exocyst complex component 3-like 4; F2RL3: F2R-like thrombin/trypsin receptor 3; FAM53B: Family with sequence similarity 53 member B; FDR: False discovery rate; FGFRL 1: Fibroblast growth factor receptor-like 1; FOXK2: Forkhead box K2; GALNT2: Polypeptide N-acetylgalactosaminyltransferase 2; GEO: Gene Expression Omnibus; GFl1: Growth factor independent 1 transcriptional repressor; GL/4: GLI family zinc finger 4; GNA12: G protein subunit alpha 12; GNG12: G protein subunit gamma 12; GNG7: G protein subunit gamma 7; GPR15: G proteincoupled receptor 15; GRK5: G protein-coupled receptor kinase 5; HLADPB1: Major histocompatibility complex, class II, DP beta 1; IER3: Immediate early response 3; IFFO1: Intermediate filament family orphan 1; INSIG 1: Insulininduced gene 1; IQR: Interquartile range; IRF7: Interferon regulatory factor 7; JAML (alias AMICA1): Junction adhesion molecule-like; KIAA0182: Gse1 coiled-coil protein; KIAA1549L (alias C11orf41): KIAA1549-like; KRBOX1: KRAB box domain containing 1; KRT7: Keratin 7; LAIR1: Leukocyte-associated immunoglobulin-like receptor 1; LDLRAD4 (alias C180rf1): Low density lipoprotein receptor class A domain containing 4; LG/1: Leucine-rich glioma inactivated 1; LGMN: Legumain; limma: Linear Models for Microarray data; LINC01019: Long intergenic nonprotein coding RNA 1019; LPCAT1: Lysophosphatidylcholine acyltransferase 1; LPP: LIM domain containing preferred translocation partner in lipoma; LY6G6E: Lymphocyte antigen 6 complex, locus G6E; MAN2B1: Mannosidase alpha class 2B member 1; MGP: Matrix Gla protein; MIR155HG: MIR155 host gene; MXRA8: Matrix remodeling associated 8; MYO1G: Myosin IG; NEAT1: Nuclear paraspeckle assembly transcript 1 (non-protein coding); NHEDC1: Solute carrier family 9 member B1; NKX2-3: NK2 homeobox 3; NT5C1A: 5'-nucleotidase, cytosolic IA; NTN1: Netrin 1; ODF3B: Outer dense fiber of sperm tails 3B; PAX8: Paired box 8; PCGF3: Polycomb group ring finger 3; PLEKHA8: Pleckstrin homology domain containing A8; PRDM8: PR/SET domain 8; PRR25: Proline-rich 25; qRT-PCR: Quantitative real-time reverse transcription polymerase chain reaction; RIN3: Ras and Rab interactor 3; RP11474D1.3: Retinitis pigmentosa 11; SATB2: SATB homeobox 2;

SCCPDH: Saccharopine dehydrogenase; SD: Standard deviation; SHISA8: Shisa family member 8; SLC16A12: Solute carrier family 16 member 12; SLFN12L: Schlafen family member 12-like; SMCO1 (alias C3orf43): Single-pass membrane protein with coiled-coil domains 1; SNCG: Synuclein gamma; SNED1: Sushi-, nidogen-, and EGF-like domains 1; SOX30: SRY-box 30; SPAG 17: Sperm-associated antigen 17; STX2: Syntaxin 2; SYNGAP1: Synaptic Ras GTPase activating protein 1; SYNGR1: Synaptogyrin 1; TBCD: Tubulin folding cofactor D; THBS2: Thrombospondin 2; TIAM2: T cell lymphoma invasion and metastasis 2; TLE3: Transducin-like enhancer of split 3; TRAPPC9: Trafficking protein particle complex 9; TRG-AS1: T cell receptor gamma locus antisense RNA 1; TSPAN13: Tetraspanin 13; UTRN: Utrophin; ZBTB38: Zinc finger and BTB domain containing 38; ZC3H12D: Zinc finger CCCH-type containing 12D; ZNF385A: Zinc finger protein 385A; ZNF697: Zinc finger protein 697;

\section{Acknowledgements}

We appreciate all of the study participants for their contribution to this research. We thank Drs. Shuangshuang Dai, Tianyuan Wang, and Sarah Reese of NIEHS and Jianping Jin of Westat, Inc. for expert computational assistance.

\section{Funding}

This research was supported by Basic Science Research Program through the National Research Foundation of Korea (NRF) funded by the Ministry of Science, ICT and Future Planning (2013R1A1A1057961), the Ministry of Education, Science and Technology (NRF-355-2011-1-E00060, NRF2012R1A6A3A01039450), the Ministry of Education (2013R1A6A3A04059017), and grants from the Environmental Health Center funded by the Ministry of Environment, Republic of Korea. This study was also supported in part by the Intramural Research Program of the NIH, National Institute of Environmental Health Sciences (NIEHS). 


\section{Availability of data and materials}

The results of epigenome-wide association study of current versus never smoking using Infinium HumanMethylation450 BeadChip are provided in Additional file 3: Table S1 of this manuscript.

\section{Authors' contributions}

WJK and YH have designed the cohort study. SJL and SYK advised analytic approach. MKL analyzed the data and wrote the manuscript draft. All authors have read and approved the final manuscript.

\section{Competing interests}

The authors declare that they have no competing interests.

\section{Consent for publication}

Not applicable.

\section{Ethics approval and consent to participate}

The Institute Review Board of the Kangwon National University Hospital approved analyses of the clinical and imaging data (Institutional Review Board of Kangwon National University Hospital 2012-06-007-001 and KNUH2016-05-003-001). Individual informed written consent was obtained from all participants. The study adhered to the tenets of the Helsinki Declaration of 1975, as revised in 2008.

\section{Author details}

'Epidemiology Branch, National Institute of Environmental Health Sciences, Department of Health and Human Services, National Institutes of Health, Research Triangle Park, Durham, NC 27709, USA. Institute of Medical Science, Kangwon National University, Chuncheon-si, Gangwon-do 24341, South Korea. ${ }^{3}$ Department of Internal Medicine and Environmental Health Center, Kangwon National University Hospital, School of Medicine, Kangwon National University, Chuncheon-si, Gangwon-do 19300, South Korea. ${ }^{4}$ Institute of Health and Environment, Seoul National University, Seoul 08826, South Korea.

Received: 18 May 2016 Accepted: 9 September 2016

Published online: 22 September 2016

\section{References}

1. Ezzati M, Lopez AD. Estimates of global mortality attributable to smoking in 2000. Lancet. 2003;362:847-52.

2. OECD: Daily smokers (indicator). https://data.oecd.org/healthrisk/dailysmokers.htm. Accessed 18 Feb 2016.

3. Vineis $P$, Alavanja M, Buffler P, Fontham E, Franceschi S, Gao YT, Gupta PC, Hackshaw A, Matos E, Samet J, et al. Tobacco and cancer: recent epidemiological evidence. J Natl Cancer Inst. 2004;96:99-106.

4. Cunningham TJ, Ford ES, Rolle IV, Wheaton AG, Croft JB. Associations of selfreported cigarette smoking with chronic obstructive pulmonary disease and co-morbid chronic conditions in the United States. COPD. 2015;12:276-86.

5. Conen D, Everett BM, Kurth T, Creager MA, Buring JE, Ridker PM, Pradhan AD. Smoking, smoking cessation, [corrected] and risk for symptomatic peripheral artery disease in women: a cohort study. Ann Intern Med. 2011; 154:719-26.

6. Goldberg $A D$, Allis $C D$, Bernstein E. Epigenetics: a landscape takes shape. Cell. 2007;128:635-8.

7. Fraga MF, Ballestar E, Paz MF, Ropero S, Setien F, Ballestar ML, Heine-Suner D, Cigudosa JC, Urioste M, Benitez J, et al. Epigenetic differences arise during the lifetime of monozygotic twins. Proc Natl Acad Sci U S A. 2005; 102:10604-9.

8. El-Maarri O, Becker T, Junen J, Manzoor SS, Diaz-Lacava A, Schwaab R, Wienker T, Oldenburg J. Gender specific differences in levels of DNA methylation at selected loci from human total blood: a tendency toward higher methylation levels in males. Hum Genet. 2007;122:505-14.

9. Dick KJ, Nelson CP, Tsaprouni L, Sandling JK, Aissi D, Wahl S, Meduri E, Morange PE, Gagnon F, Grallert $H$, et al. DNA methylation and body-mass index: a genome-wide analysis. Lancet. 2014;383:1990-8.

10. Lee KW, Pausova Z. Cigarette smoking and DNA methylation. Front Genet. 2013;4:132.

11. Allione A, Marcon F, Fiorito G, Guarrera S, Siniscalchi E, Zijno A, Crebelli R, Matullo G. Novel epigenetic changes unveiled by monozygotic twins discordant for smoking habits. PLoS One. 2015;10:1-11.
12. Guida F, Sandanger TM, Castagne R, Campanella G, Polidoro S, Palli D, Krogh V, Tumino R, Sacerdote C, Panico S, et al. Dynamics of smokinginduced genome-wide methylation changes with time since smoking cessation. Hum Mol Genet. 2015;24:2349-59.

13. Zaghlool SB, Al-Shafai M, Al Muftah WA, Kumar P, Falchi M, Suhre K. Association of DNA methylation with age, gender, and smoking in an Arab population. Clin Epigenetics. 2015;7:6.

14. Besingi W, Johansson A. Smoke-related DNA methylation changes in the etiology of human disease. Hum Mol Genet. 2014;23:2290-7.

15. Dogan MV, Shields B, Cutrona C, Gao L, Gibbons FX, Simons R, Monick M, Brody GH, Tan K, Beach SR, Philibert RA. The effect of smoking on DNA methylation of peripheral blood mononuclear cells from African American women. BMC Genomics. 2014;15:151.

16. Elliott HR, Tillin T, McArdle WL, Ho K, Duggirala A, Frayling TM, Davey Smith G, Hughes AD, Chaturvedi N, Relton CL. Differences in smoking associated DNA methylation patterns in South Asians and Europeans. Clin Epigenetics. 2014;6:4.

17. Harlid S, Xu Z, Panduri V, Sandler DP, Taylor JA. CpG sites associated with cigarette smoking: analysis of epigenome-wide data from the sister study. Environ Health Perspect. 2014.

18. Tsaprouni LG, Yang TP, Bell J, Dick KJ, Kanoni S, Nisbet J, Vinuela A, Grundberg E, Nelson CP, Meduri E, et al. Cigarette smoking reduces DNA methylation levels at multiple genomic loci but the effect is partially reversible upon cessation. Epigenetics. 2014;9:1382-96.

19. Shenker NS, Polidoro S, van Veldhoven K, Sacerdote C, Ricceri F, Birrell MA, Belvisi MG, Brown R, Vineis P, Flanagan JM. Epigenome-wide association study in the European Prospective Investigation into Cancer and Nutrition (EPIC-Turin) identifies novel genetic loci associated with smoking. Hum Mol Genet. 2013;22:843-51.

20. Sun YV, Smith AK, Conneely KN, Chang Q, Li W, Lazarus A, Smith JA, Almli LM, Binder EB, Klengel $T$, et al. Epigenomic association analysis identifies smokingrelated DNA methylation sites in African Americans. Hum Genet. 2013.

21. Philibert RA, Beach SR, Lei MK, Brody GH. Changes in DNA methylation at the aryl hydrocarbon receptor repressor may be a new biomarker for smoking. Clin Epigenetics. 2013;5:19.

22. Zeilinger S, Kuhnel B, Klopp N, Baurecht H, Kleinschmidt A, Gieger C, Weidinger S, Lattka E, Adamski J, Peters A, et al. Tobacco smoking leads to extensive genome-wide changes in DNA methylation. PLoS One. 2013;8:e63812.

23. Philibert RA, Beach SR, Brody GH. Demethylation of the aryl hydrocarbon receptor repressor as a biomarker for nascent smokers. Epigenetics. 2012;7:1331-8.

24. Wan ES, Qiu W, Baccarelli A, Carey VJ, Bacherman H, Rennard SI, Agusti A, Anderson W, Lomas DA, Demeo DL. Cigarette smoking behaviors and time since quitting are associated with differential DNA methylation across the human genome. Hum Mol Genet. 2012;21:3073-82.

25. Breitling LP, Yang R, Korn B, Burwinkel B, Brenner H. Tobacco-smokingrelated differential DNA methylation: $27 \mathrm{~K}$ discovery and replication. Am J Hum Genet. 2011:88:450-7.

26. Zhu X, Li J, Deng S, Yu K, Liu X, Deng Q, Sun H, Zhang X, He M, Guo H, et al. Genome-wide analysis of DNA methylation and cigarette smoking in Chinese. Environ Health Perspect. 2016.

27. Zhang Y, Florath I, Saum KU, Brenner H. Self-reported smoking, serum cotinine, and blood DNA methylation. Environ Res. 2016;146:395-403.

28. Lister R, Pelizzola M, Dowen RH, Hawkins RD, Hon G, Tonti-Filippini J, Nery $J R$, Lee L, Ye Z, Ngo QM, et al. Human DNA methylomes at base resolution show widespread epigenomic differences. Nature. 2009:462:315-22.

29. Jaffe $A E$, Murakami $P$, Lee $H$, Leek JT, Fallin MD, Feinberg AP, Irizarry RA. Bump hunting to identify differentially methylated regions in epigenetic epidemiology studies. Int J Epidemiol. 2012;41:200-9.

30. Kim WJ, Lim JH, Lee JS, Lee SD, Kim JH, Oh YM. Comprehensive analysis of transcriptome sequencing data in the lung tissues of COPD subjects. Int J Genomics. 2015;2015:206937.

31. Yoonki Hong J-WK, Lee S-A, Young JH, Moon JY, Kim HY, Han S-S, Lee S-J, Kim WJ. Methdology of an observational cohort study for subjects with chronic obstructive pulmonary disease in dusty areas near cement plants. J Pulm Respir Med. 2014;04:169-74.

32. Gorber SC, Schofield-Hurwitz S, Hardt J, Levasseur G, Tremblay M. The accuracy of self-reported smoking: a systematic review of the relationship between self-reported and cotinine-assessed smoking status. Nicotine Tobacco Res. 2009;11:12-24.

33. Teschendorff AE, Marabita F, Lechner M, Bartlett T, Tegner J, Gomez-Cabrero D, Beck S. A beta-mixture quantile normalization method for correcting 
probe design bias in Illumina Infinium 450k DNA methylation data. Bioinformatics. 2013;29:189-96.

34. Morris TJ, Butcher LM, Feber A, Teschendorff AE, Chakravarthy AR, Wojdacz TK, Beck S. ChAMP: 450k chip analysis methylation pipeline. Bioinformatics. 2014;30:428-30.

35. Johnson WE, Li C, Rabinovic A. Adjusting batch effects in microarray expression data using empirical Bayes methods. Biostatistics. 2007;8:1 18-27.

36. Houseman EA, Accomando WP, Koestler DC, Christensen BC, Marsit CJ, Nelson HH, Wiencke JK, Kelsey KT. DNA methylation arrays as surrogate measures of cell mixture distribution. BMC Bioinformatics. 2012;13:86.

37. Aryee MJ, Jaffe AE, Corrada-Bravo H, Ladd-Acosta C, Feinberg AP, Hansen $K D$, Irizarry RA. Minfi: a flexible and comprehensive bioconductor package for the analysis of infinium DNA methylation microarrays. Bioinformatics. 2014;30:1363-9.

38. Morris TJ, Beck S. Analysis pipelines and packages for Infinium HumanMethylation450 Bead Chip (450k) data. Methods. 2015;72:3-8.

39. Zhang $X, M u ~ W$, Zhang $W$. On the analysis of the illumina 450k array data: probes ambiguously mapped to the human genome. Front Genet. 2012;3:73.

40. Marabita F, Almgren M, Lindholm ME, Ruhrmann S, Fagerstrom-Billai F Jagodic M, Sundberg CJ, Ekstrom TJ, Teschendorff AE, Tegner J, GomezCabrero D. An evaluation of analysis pipelines for DNA methylation profiling using the Illumina HumanMethylation450 BeadChip platform. Epigenetics. 2013;8:333-46

41. Du P, Zhang X, Huang CC, Jafari N, Kibbe WA, Hou L, Lin SM. Comparison of beta-value and M-value methods for quantifying methylation levels by microarray analysis. BMC Bioinformatics. 2010;11:587.

42. Smyth GK. Linear models and empirical Bayes methods for assessing differential expression in microarray experiments. Stat Appl Genet Mol Biol. 2004;3:Article 3.

43. Benjamini Y, Hochberg Y. Controlling the false discovery rate-a practical and powerful approach to multiple testing. J Roy Statist Soc Ser B. 1995;57:289-300.

44. Peters TJ, Buckley MJ, Statham AL, Pidsley R, Samaras K, V Lord R, Clark SJ, Molloy PL. De novo identification of differentially methylated regions in the human genome. Epigenetics Chromatin. 2015;8:6.

45. Pedersen BS, Schwartz DA, Yang IV, Kechris KJ. Comb-p: software for combining, analyzing, grouping and correcting spatially correlated P-values. Bioinformatics. 2012;28:2986-8.

46. Satterthwaite FE. An approximate distribution of estimates of variance components. Biometrics. 1946;2:110-4.

47. Sidak Z. Rectangular confidence regions for the means of multivariate normal distributions. J Am Stat Assoc. 1967:62:8.

48. Package 'DMRcate' [https://www.bioconductor.org/packages/release/bioc/ manuals/DMRcate/man/DMRcate.pdf]. Accessed 10 Mar 2016.

49. Yang IV, Pedersen BS, Liu A, O'Connor GT, Teach SJ, Kattan M, Misiak RT, Gruchalla R, Steinbach SF, Szefler SJ, et al. DNA methylation and childhood asthma in the inner city. J Allergy Clin Immunol. 2015;136:69-80.

50. Computing RFS. R: A language and environment for statistical computing Vienna: R Core Team; 2013.

51. Illumina Infinium HumanMethylation450 BeadChip Annotation. https:// support.illumina.com/array/array_kits/infinium_humanmethylation450_ beadchip_kit/downloads.html. Accessed 18 Sept 2015.

52. Martin TC, Yet I, Tsai PC, Bell JT. coMET: visualisation of regional epigenomewide association scan results and DNA co-methylation patterns. BMC Bioinformatics. 2015;16:131.

53. Kuo HW, Huang CY, Fu CK, Liao CH, Hsieh YH, Hsu CM, Tsai CW, Chang WS, Bau DT. The significant association of CCND1 genotypes with gastric cancer in Taiwan. Anticancer Res. 2014;34:4963-8.

54. Shih LC, Tsai CW, Tsai MH, Tsou YA, Chang WS, Li FJ, Lee MH, Bau DT. Association of cyclin D1 genotypes with nasopharyngeal carcinoma risk. Anticancer Res. 2012;32:1093-8.

55. Sobti RC, Kaur P, Kaur S, Singh J, Janmeja AK, Jindal SK, Kishan J, Raimondi S. Effects of cyclin D1 (CCND1) polymorphism on susceptibility to lung cancer in a North Indian population. Cancer Genet Cytogenet. 2006;170:108-14.

56. Hsia TC, Liu CJ, Lin CH, Chang WS, Chu CC, Hang LW, Lee HZ, Lo WC, Bau DT. Interaction of CCND1 genotype and smoking habit in Taiwan lung cancer patients. Anticancer Res. 2011;31:3601-5.

57. Zhang Y, Yang RX, Burwinkel B, Breitling LP, Brenner H. F2RL3 methylation as a biomarker of current and lifetime smoking exposures. Environ Health Perspect. 2014;122:131-7.
58. Zhang Y, Schottker B, Florath I, Stock C, Butterbach K, Holleczek B, Mons U, Brenner H. Smoking-Associated DNA Methylation Biomarkers and Their Predictive Value for All-Cause and Cardiovascular Mortality. Environ Health Perspect. 2016;124:67-74.

59. Lin FY, Lin CW, Yang SF, Lee WJ, Lin YW, Lee LM, Chang JL, Weng WC, Lin $\mathrm{CH}$, Chien $\mathrm{MH}$. Interactions between environmental factors and melatonin receptor type $1 \mathrm{~A}$ polymorphism in relation to oral cancer susceptibility and clinicopathologic development. PLoS One. 2015;10.

60. Ziller MJ, Gu H, Muller F, Donaghey J, Tsai LT, Kohlbacher O, De Jager PL, Rosen ED, Bennett DA, Bernstein BE, et al. Charting a dynamic DNA methylation landscape of the human genome. Nature. 2013;500:477-81.

61. Rebagliato M. Validation of self reported smoking. J Epidemiol Community Health. 2002:56:163-4.

\section{Submit your next manuscript to BioMed Central and we will help you at every step:}

- We accept pre-submission inquiries

- Our selector tool helps you to find the most relevant journal

- We provide round the clock customer support

- Convenient online submission

- Thorough peer review

- Inclusion in PubMed and all major indexing services

- Maximum visibility for your research

Submit your manuscript at www.biomedcentral.com/submit
Biomed Central 